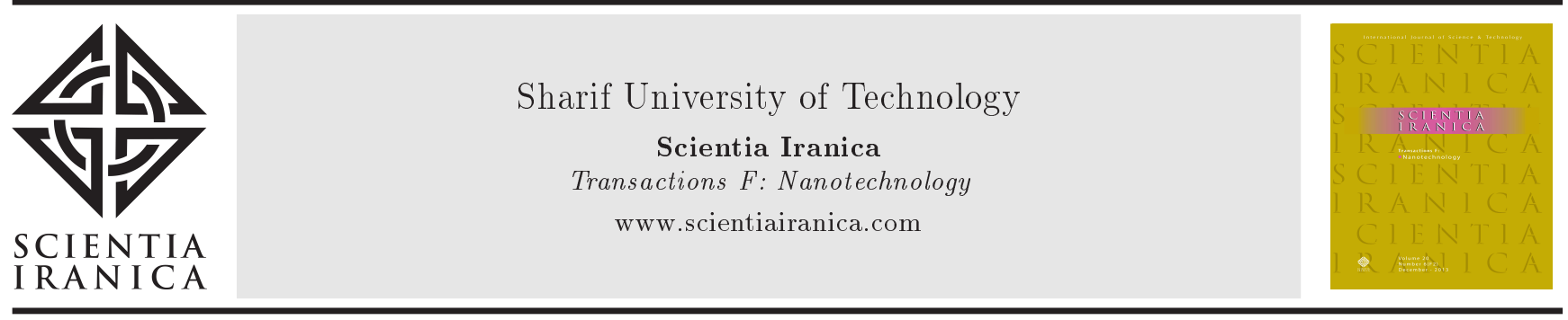

\title{
Nanofluid effects on peristaltic transport of a fourth grade fluid in the occurrence of inclined magnetic field
}

\author{
S. Akram* \\ Department of Basic Sciences, MCS, National University of Sciences and Technology (NUST), Islamabad, 44000, Pakistan.
}

Received 22 November 2014; received in revised form 28 March 2015; accepted 16 June 2015

\section{KEYWORDS}

Peristaltic flow;

Nanofluid particles;

Fourth grade fluid;

Inclined magnetic

field;

Asymmetric channel;

Different wave forms.

\begin{abstract}
This paper deals with the effect of nanofluid and inclined magnetic field on peristaltic transport of a fourth grade fluid model. Mathematical modelling of two dimensional fourth grade fluid along with nanofluid model are given for channel. Assumptions of long wave length are employed to simplify the governing equations of momentum, temperature, and nano particle volume fraction. The exact solutions of temperature and nano particle volume fraction are calculated. Analytical solution is carried out to calculate the solution of stream function. Pressure rise and pressure gradient on the channel walls have been computed numerically. The graphical results are discussed to see the effects of various emerging parameters on different wave forms.
\end{abstract}

(C) 2016 Sharif University of Technology. All rights reserved.

\section{Introduction}

Nowadays, the study of peristaltic flow has received a considerable attention from many researchers due to its wide range of applications in engineering, industry, and physiology. Peristaltic flows basically occur in swallowing food through the esophagus, urine transport from kidney to bladder, lymph transport in the lymphatic vessels, vasomotion of small blood vessels, cell separators, arthropumps, artificial blood pumps and heart-lung machine, finger and roller pumps, toxic liquid in nuclear industry, etc. In literature, sundry works concerning peristaltic motion have been done for Newtonian fluid and non-Newtonian fluids with different flow geometries [1-8]. The seminal work done is given by Latham [9] and Shapiro et al. [10].

Recently, research on MHD flow problems has attained important interest due to its wide-ranging use in engineering and medical applications. Magnetic wound or cancer tumor treatment causing hyperthermia, bleeding reduction during surgeries, and targeted

*.E-mail addresses: drsafiaakram@gmail.com and safia_akram@yahoomail.com transport of drugs using magnetic particles as drug carriers are few such examples. Recently, Akram and Nadeem [11] discussed the induced magnetic field and heat transfer on the peristaltic motion of a Jeffrey fluid model. An effect of magnetic field on peristaltic flow in a non-uniform channel was investigated in [12]. Mekheimer et al. [13] have discussed nonlinear peristaltic transport of MHD flow through a porous medium. An effect of inclined magnetic field on magneto fluid flow through porous medium between two inclined wavy porous plates was explored in [14]. A thorough discussion on the MHD peristaltic flows under various assumptions has been available in the studies [15-18].

Another important area of study is the nanofluid which has newly fascinated the attention of many researchers. A nanofluid is a fluid including nanometersized particles, called nanoparticles. Nanofluids have unique properties that make them potentially useful in many applications in heat transfer [19], including microelectronics, fuel cells, pharmaceutical processes, engine cooling/vehicle thermal management, domestic refrigerator, chiller, heat exchanger, nuclear reactor coolant, grinding, machining, space technology, defense 
and ships, and boiler flue gas temperature reduction. The pioneer work done on nanofluid was by Choi [20]. Khan and Pop [21] studied boundarylayer flow of nanofluid over a stretching sheet. Heat transfer performance of a nanofluid inside an enclosure pool was examined by Khanafer et al. [22]. There exist extensive studies on nanofluids of viscous and non-Newtonian fluids [23-33]. Recently, Akram and Nadeem [34] discussed the effects of nanofluid and partial slip on the peristaltic transport of a Jeffrey fluid model in an asymmetric channel. In another article, nanofluid effects on peristaltic transport of a hyperbolic tangent fluid model in the presence of apt (tending) magnetic field was studied by Akram and Nadeem [35].

Motivated by the above discussion, the aim of the present paper is to discuss the effects of nanofluid on peristaltic transport of a fourth grade fluid in an asymmetric channel with different wave forms. To our knowledge, in literature, no material is available to discuss the effects of nanofluids on a peristaltic flow of a fourth grade fluid in an asymmetric channel. The remainder of the paper is organized into the following major sections: Section 2 describes the general form of the governing equations of the present flow problem. Section 3 illustrates the mathematical development of the flow under consideration while Section 4 describes the solution of the highly non-linear coupled equations. Section 5 deals with the numerical and graphical results of the present flow problem.

\section{Governing equations}

The balance of mass and momentum for an incompressible fluid is given by:

$$
\begin{aligned}
& \operatorname{div} \mathbf{V}=0, \\
& \rho \frac{d \mathbf{V}}{d t}=\operatorname{div} \mathbf{T}_{1}+\rho \mathbf{f}, \\
&(\rho c)_{f} \frac{d \mathbf{T}}{d t}=K \nabla^{2} \mathbf{T}+(\rho c)_{\rho}\left(D_{B} \nabla \mathbf{C} \nabla \mathbf{T}+\left(\frac{D_{T}}{T_{0}}\right) \nabla \mathbf{T} \nabla \mathbf{T}\right), \\
& \frac{d \mathbf{C}}{d t}=D_{B} \nabla^{2} \mathbf{C}+\left(\frac{D_{T}}{T_{0}}\right) \nabla^{2} \mathbf{T},
\end{aligned}
$$

where $\rho$ is the density, $\mathbf{V}$ is the velocity vector, $\mathbf{T}_{1}$ is the Cauchy stress tensor, $\mathbf{f}$ represents the specific body force, and $d / d t$ represents the material time derivative. The constitutive equation for the fourth grade fluid is given by [36]:

$$
\mathbf{T}_{1}=-P \mathbf{I}+\mathbf{S}
$$

$$
\begin{aligned}
\mathbf{S}= & \mu A_{1}+\alpha_{1} A_{2}+\alpha_{2} A_{1}^{2}+\beta_{1} A_{3} \\
& +\beta_{2}\left(A_{1} A_{2}+A_{2} A_{1}\right)+\beta_{3}\left(\operatorname{tr} A_{1}^{2}\right) A_{1}+\gamma_{1} A_{4} \\
& +\gamma_{2}\left(A_{1} A_{3}+A_{3} A_{1}\right)+\gamma_{3} A_{2}^{2} \\
& +\gamma_{4}\left(A_{1}^{2} A_{2}+A_{2} A_{1}^{2}\right)+\gamma_{5} \operatorname{trac}\left(A_{2}\right) A_{2} \\
& +\gamma_{6} \operatorname{trac}\left(A_{2}\right) A_{1}^{2}+\left(\gamma_{7} \operatorname{trac} A_{3}\right. \\
& \left.+\gamma_{8} \operatorname{trac} A_{2} A_{1}\right) A_{1}, \\
A_{1}= & (\operatorname{grad} \mathbf{V})+(\operatorname{grad} \mathbf{V})^{T}, \\
A_{n}= & \frac{d A_{n-1}}{d t}+A_{n-1}(\operatorname{grad} \mathbf{V})+(\operatorname{grad} \mathbf{V})^{T} A_{n-1},
\end{aligned}
$$

where $\mu$ is the constant viscosity, $\alpha_{1}, \alpha_{2}, \beta_{1}-\beta_{3}, \gamma_{1}-$ $\gamma_{8}$ are the material constants, and $A_{n}$ is the RivilinEricksen tensors.

\section{Mathematical formulation}

Let us consider an incompressible peristaltic flow of a fourth grade fluid. The flow is generated by sinusoidal wave trains propagating with constant speed $c$ along the channel walls. We also consider that the magnetic field is inclined at an angle $\Theta$. The lower and upper walls of the channel are maintained at constant temperatures $T_{0}$ and $T_{1}$, and at constant concentrations $C_{0}$ and $C_{1}$, respectively.

We seek the velocity field for the two-dimensional and two-directional flow of the form:

$$
\mathbf{V}=(U(X, Y, t), V(X, Y, t), 0)
$$

For the two-dimensional incompressible flow, the governing equations of motion, including nanofluid and inclined magnetic field, in the laboratory frame $(X, Y)$ can be written as:

$$
\begin{aligned}
& \frac{\partial U}{\partial X}+\frac{\partial V}{\partial Y}=0 \\
& \rho_{f}\left(\frac{\partial U}{\partial t}+U \frac{\partial U}{\partial X}+V \frac{\partial U}{\partial Y}\right)=-\frac{\partial P}{\partial X}+\frac{\partial}{\partial X}\left(S_{X X}\right) \\
& \quad+\frac{\partial}{\partial Y}\left(S_{X Y}\right)-\sigma B_{0}^{2} \cos \Theta(U \cos \Theta-V \sin \Theta) \\
& \quad+\rho g \alpha\left(T-T_{0}\right)+\rho g \alpha\left(C-C_{0}\right) \\
& \rho_{f}\left(\frac{\partial V}{\partial t}+U \frac{\partial V}{\partial X}+V \frac{\partial V}{\partial Y}\right)=-\frac{\partial P}{\partial Y}+\frac{\partial}{\partial X}\left(S_{Y X}\right) \\
& \quad+\frac{\partial}{\partial Y}\left(S_{Y Y}\right)+\sigma B_{0}^{2} \sin \Theta(U \cos \Theta-V \sin \Theta)
\end{aligned}
$$




$$
\begin{gathered}
\left(\frac{\partial T}{\partial t}+U \frac{\partial T}{\partial X}+V \frac{\partial T}{\partial Y}\right)=\alpha\left(\frac{\partial^{2} T}{\partial X^{2}}+\frac{\partial^{2} T}{\partial Y^{2}}\right) \\
+\tau\left\{D_{B}\left(\frac{\partial C}{\partial X} \frac{\partial T}{\partial X}+\frac{\partial C}{\partial Y} \frac{\partial T}{\partial Y}\right)\left(\frac{D_{T}}{T_{0}}\right)\right. \\
\left.\left[\left(\frac{\partial T}{\partial X}\right)^{2}+\left(\frac{\partial T}{\partial Y}\right)^{2}\right]\right\}, \\
\left(\frac{\partial C}{\partial t}+U \frac{\partial C}{\partial X}+V \frac{\partial C}{\partial Y}\right)=D_{B}\left(\frac{\partial^{2} C}{\partial X^{2}}+\frac{\partial^{2} C}{\partial Y^{2}}\right) \\
+\left(\frac{D_{T}}{T_{0}}\right)\left(\frac{\partial^{2} T}{\partial X^{2}}+\frac{\partial^{2} T}{\partial Y^{2}}\right),
\end{gathered}
$$

where $U$ and $V$ are the velocities in $X$ and $Y$ directions in the fixed frame, $\rho_{f}$ is density of fluid base, $P$ is pressure, $S_{X X}, S_{X Y}$ and $S_{Y Y}$ are components of extra stress tensor, $B_{0}$ is the constant magnetic field, $g$ is the acceleration due to gravity, $T$ is temperature, $D_{B}$ is the Brownian diffusion coefficient, $D_{T}$ is the thermophoretic diffusion coefficient, $\tau=\frac{(\rho c)_{p}}{(\rho c)_{f}}$ is the ratio of the effective heat capacity of the nanoparticle material and heat capacity of the fluid with $\rho$ being the density, $c$ is the volumetric volume expansion coefficient, and $\rho_{p}$ is the density of particles.

Since in the laboratory frame $(X, Y)$ the flow is unsteady, in a coordinate system moving with the wave speed $c$ (the wave frame), the motion is steady. The coordinates and velocities in two frames are related by the following transformation:

$$
\begin{aligned}
& x=X-c t, \quad y=Y, \quad u=U-c, \\
& v=V, \quad \text { and } \quad p(x, y)=P(X, Y, t) .
\end{aligned}
$$

Defining the following non-dimensional quantities:

$$
\begin{array}{ll}
\bar{x}=\frac{x}{\lambda}, & \bar{y}=\frac{y}{d_{1}}, \\
\bar{u}=\frac{u}{c}, & \bar{v}=\frac{v}{c}, \\
\delta=\frac{a}{\lambda}, & \bar{p}=\frac{d^{2} p}{\mu c \lambda}, \\
\bar{t}=\frac{c t}{\lambda}, & \operatorname{Re}=\frac{c d}{v}, \\
\bar{\Psi}=\frac{\Psi}{c d_{1}}, & \theta=\frac{T-T_{0}}{T_{1}-T_{0}}, \\
\bar{S}=\frac{a}{\mu c} S, & M=\sqrt{\frac{\sigma}{v}} B_{0} a, \\
\alpha=\frac{K}{(c \rho)_{f}}, & \operatorname{Pr}=\frac{\nu}{\alpha},
\end{array}
$$

$$
\begin{aligned}
& N_{t}=\frac{\tau D_{T}\left(T_{1}-T_{0}\right)}{T_{0} \nu}, \\
& N_{b}=\frac{\tau D_{B}\left(C_{1}-C_{0}\right)}{v}, \\
& \mathrm{Gr}=\frac{\rho g \alpha a^{2}\left(T_{1}-T_{0}\right)}{\mu c}, \\
& \mathrm{Br}=\frac{\rho g \alpha a^{2}\left(C_{1}-C_{0}\right)}{\mu c}, \\
& \mathrm{Le}=\frac{v}{D_{B}}, \\
& \lambda_{i}=\frac{\alpha_{i} c}{\mu a}(i=1,2), \\
& \xi_{i}=\frac{\beta_{i} c^{2}}{\mu a^{2}}(i=1,2,3), \\
& \eta_{i}=\frac{\gamma_{i} c^{3}}{\mu a^{3}}(i=1-8) .
\end{aligned}
$$

Using Eqs. (14) and (15), the resulting equations in terms of stream function $\Psi$ (dropping the bars, $u=\frac{\partial \Psi}{\partial y}$, $\left.v=-\delta \frac{\partial \Psi}{\partial x}\right)$ can be written as:

$$
\begin{gathered}
\operatorname{Re} \delta\left(\Psi_{y} \Psi_{x y}-\Psi_{x} \Psi_{y y}\right)=-\frac{\partial p}{\partial x}+\delta \frac{\partial}{\partial x}\left(S_{x x}\right) \\
+\frac{\partial}{\partial y}\left(S_{x y}\right)+\operatorname{Gr} \theta+B_{r} \Phi \\
-M^{2} \cos \Theta\left(\left(\Psi_{y}+1\right) \cos \Theta+\delta \Psi_{x} \sin \Theta\right), \\
\operatorname{Re} \delta^{3}\left(-\Psi_{y} \Psi_{x x}+\Psi_{x} \Psi_{x y}\right)=-\frac{\partial p}{\partial y}+\delta \frac{\partial}{\partial x}\left(S_{y x}\right) \\
+\delta \frac{\partial}{\partial y}\left(S_{y y}\right)+M^{2} \delta \sin \Theta\left(\left(\Psi_{y}+1\right) \cos \Theta\right. \\
\left.+\delta \Psi_{x} \sin \Theta\right), \\
\operatorname{Re} \delta\left(\Psi_{y} \theta_{x}-\Psi_{x} \theta_{y}\right)=\frac{1}{\operatorname{Pr}}\left(\theta_{y y}+\delta^{2} \theta_{x x}\right) \\
+N_{b}\left(\delta^{2} \theta_{x} \Phi_{x}+\theta_{y} \Phi_{y}\right) \\
+N_{t}\left(\delta^{2}\left(\theta_{x}\right)^{2}+\left(\theta_{y}\right)^{2}\right), \\
\operatorname{Re} \delta \operatorname{Le}\left(\Psi_{y} \Phi_{x}-\Psi_{x} \Phi_{y}\right)=\left(\Phi_{y y}+\delta^{2} \Phi_{x x}\right) \\
+\delta^{2} \frac{N_{t}}{N_{b}} \theta_{x x}+\frac{N_{t}}{N_{b}} \theta_{y y} .
\end{gathered}
$$

The values of $S_{x x}, S_{x y}$, and $S_{y y}$ can be computed using Eqs. (5) to (8). Hence, under the assumption of long 
wave length $\delta \ll 1$ and low Reynolds number, Eqs. (16) to (19) become:

$$
\begin{aligned}
& 0=-\frac{\partial p}{\partial x}+\frac{\partial}{\partial y} S_{x y}-M^{2} \cos ^{2} \Theta\left(\Psi_{y}+1\right) \\
&+\operatorname{Gr} \theta+B_{r} \Phi \\
& 0=-\frac{\partial p}{\partial y} \\
& \frac{1}{\operatorname{Pr}} \frac{\partial^{2} \theta}{\partial y^{2}}+N_{b} \frac{\partial \theta}{\partial y} \frac{\partial \Phi}{\partial y}+N_{t}\left(\frac{\partial \theta}{\partial y}\right)^{2}=0, \\
& \frac{\partial^{2} \Phi}{\partial y^{2}}+\frac{N_{t}}{N_{b}} \frac{\partial^{2} \theta}{\partial y^{2}}=0 .
\end{aligned}
$$

Elimination of pressure from Eqs. (20) and (21) yields:

$$
\begin{aligned}
& \frac{\partial^{2}}{\partial y^{2}} S_{x y}-M^{2} \cos ^{2} \Theta \frac{\partial^{2} \Psi}{\partial y^{2}}+\operatorname{Gr} \frac{\partial \theta}{\partial y} \\
&+B_{r} \frac{\partial \Phi}{\partial y}=0
\end{aligned}
$$

where:

$$
S_{x y}=\frac{\partial^{2} \Psi}{\partial y^{2}}+2 \Gamma\left(\frac{\partial^{2} \Psi}{\partial y^{2}}\right)^{3},
$$

and $\Gamma=\xi_{2}+\xi_{3}$ is the Deborah number.

Using Eq. (25) into Eqs. (20) and (24), we get the modified form of Eqs. (20) and (24) as:

$$
\begin{aligned}
\frac{\partial^{4} \Psi}{\partial y^{4}} & +2 \Gamma \frac{\partial^{2}}{\partial y^{2}}\left(\frac{\partial^{2} \Psi}{\partial y^{2}}\right)^{3}-M^{2} \cos ^{2} \Theta \frac{\partial^{2} \Psi}{\partial y^{2}} \\
& +\operatorname{Gr} \frac{\partial \theta}{\partial y}+B_{r} \frac{\partial \Phi}{\partial y}=0 \\
\frac{d p}{d x}= & \frac{\partial}{\partial y}\left(\frac{\partial^{2} \Psi}{\partial y^{2}}+2 \Gamma\left(\frac{\partial^{2} \Psi}{\partial y^{2}}\right)^{3}\right) \\
& -M^{2} \cos ^{2} \Theta\left(\Psi_{y}+1\right)+\operatorname{Gr} \theta+B_{r} \Phi .
\end{aligned}
$$

The corresponding dimensionless boundary conditions in terms of stream function are defined as:

$$
\begin{aligned}
& \Psi=\frac{F}{2} \quad \text { at } \quad y=h_{1}=1+a \cos 2 \pi x, \\
& \Psi=-\frac{F}{2} \quad \text { at } \quad y=h_{2}=-d-b \cos (2 \pi x+\phi), \\
& \frac{\partial \Psi}{\partial y}=-1 \quad \text { at } \quad y=h_{1} \quad \text { and } \quad y=h_{2}, \\
& \theta=0, \quad \Phi=0, \quad \gamma=0 \quad \text { at } y=h_{1}, \\
& \theta=1, \quad \Phi=1, \quad \gamma=1 \quad \text { at } y=h_{2} .
\end{aligned}
$$

The dimensionless mean flow $Q$ is defined as:

$$
Q=F+1+d,
$$

where $F$ is the dimensionless time mean flow rate in the wave frame. It is related to dimensionless time mean flow rate $Q$ in the laboratory frame through the expression $Q=F+1+d$.

Since our interest in the present study is to perform the analysis for the sinusoidal, multisinusional, triangular, trapezoidal, and square waves, the expressions for triangular, square, and trapezoidal waves are derived from Fourier series.

The non-dimensional expressions for the considered wave form are given as:

1. Sinusoidal wave:

$$
\begin{aligned}
& h_{1}(x)=1+a \sin 2 \pi x, \\
& h_{2}(x)=-d-b \sin (2 \pi x+\phi) .
\end{aligned}
$$

2. Multisinusional wave:

$$
\begin{aligned}
& h_{1}(x)=1+a \sin 2 n \pi x, \\
& h_{2}(x)=-d-b \sin (2 n \pi x+\phi) .
\end{aligned}
$$

3. Triangular wave:

$$
\begin{aligned}
h_{1}(x) & =1 \\
+a & {\left[\frac{8}{\pi^{3}} \sum_{m=1}^{\infty} \frac{(-1)^{m+1}}{(2 m-1)^{2}} \sin (2 \pi(2 m-1) x)\right], } \\
h_{2}(x) & =-d \\
-b & {\left[\frac{8}{\pi^{3}} \sum_{m=1}^{\infty} \frac{(-1)^{m+1}}{(2 m-1)^{2}} \sin (2 \pi(2 m-1) x+\phi)\right] . }
\end{aligned}
$$

4. Trapezoidal wave:

$$
\begin{aligned}
& h_{1}(x)=1 \\
& +a\left[\frac{32}{\pi^{2}} \sum_{m=1}^{\infty} \frac{\sin \frac{\pi}{8}(2 m-1)}{(2 m-1)^{2}} \sin (2 \pi(2 m-1) x)\right], \\
& h_{2}(x)=-d \\
& -b\left[\frac{32}{\pi^{2}} \sum_{m=1}^{\infty} \frac{\sin \frac{\pi}{8}(2 m-1)}{(2 m-1)^{2}} \sin (2 \pi(2 m-1) x+\phi)\right] .
\end{aligned}
$$

5. Square wave:

$$
\begin{aligned}
& h_{1}(x)=1 \\
& \quad+a\left[\frac{4}{\pi} \sum_{m=1}^{\infty} \frac{(-1)^{m+1}}{(2 m-1)} \cos (2(2 m-1) \pi x)\right],
\end{aligned}
$$




$$
\begin{aligned}
h_{2}(x) & =-d \\
-b & {\left[\frac{4}{\pi} \sum_{m=1}^{\infty} \frac{(-1)^{m+1}}{(2 m-1)} \cos (2(2 m-1) \pi x+\phi)\right] . }
\end{aligned}
$$

\section{Solution of the problem}

In this fragment, a valuable procedure is described to calculate the solutions for the present system of linear and non-linear differential equations. From Eq. (23) we obtain:

$$
\Phi(x, y)=-\frac{N_{t}}{N_{b}} \theta+L_{1}(x) y+L_{2}(x),
$$

where $L_{1}(x)$ and $L_{2}(x)$ are unknown functions and can be evaluated using the boundary conditions. Now, substituting Eq. (32) into Eq. (22), we get:

$$
\frac{\partial^{2} \theta}{\partial y^{2}}+\operatorname{Pr} N_{b} L_{1}(x) \frac{\partial \theta}{\partial y}=0 .
$$

The exact solution of Eq. (33) gives the temperature distribution as:

$$
\theta(x, y)=\frac{L_{3}(x)}{\operatorname{Pr} N_{b} L_{1}(x)}+L_{4}(x) e^{-\operatorname{Pr} N_{b} L_{1}(x) y},
$$

where $L_{3}(x)$ and $L_{4}(x)$ are unknown functions. Now, with the help of temperature distribution (Eq. (34)), the nano-particle concentration $\Phi$ is given from Eq. (32) as:

$$
\begin{aligned}
\Phi(x, y)= & -\frac{N_{t}}{N_{b}}\left(\frac{L_{3}(x)}{\operatorname{Pr} N_{b} L_{1}(x)}\right. \\
& \left.+L_{4}(x) e^{-\operatorname{Pr} N_{b} L_{1}(x) y}\right)+L_{1}(x) y+L_{2}(x)
\end{aligned}
$$

Now, calculate the values of unknown functions $L_{1}(x)$, $L_{2}(x), L_{3}(x)$ and $L_{4}(x)$ by applying the boundary conditions defined as:

$$
\begin{aligned}
& L_{1}(x)=\frac{1+\frac{N_{t}}{N_{b}}}{h_{2}-h_{1}} \\
& L_{2}(x)=-h_{1}\left(\frac{1+\frac{N_{t}}{N_{b}}}{h_{2}-h_{1}}\right) \text {, } \\
& L_{3}(x)= \\
& -\operatorname{Pr} N_{b} L_{1}(x)\left(\frac{e^{-\operatorname{Pr} N_{b} L_{1}(x) h_{1}}}{e^{-\operatorname{Pr} N_{b} L_{1}(x) h_{2}}-e^{-\operatorname{Pr} N_{b} L_{1}(x) h_{1}}}\right) . \\
& L_{4}(x)=\frac{1}{e^{-\operatorname{Pr} N_{b} L_{1}(x) h_{2}}-e^{-\operatorname{Pr} N_{b} L_{1}(x) h_{1}}} .
\end{aligned}
$$

Thus, the exact expressions for the temperature distribution $\theta$ and nano-particle concentration $\Phi$ are given by:

$$
\begin{aligned}
\theta(x, y) & =\frac{e^{-\operatorname{Pr} N_{b} L_{1}(x) y}-e^{-\operatorname{Pr} N_{b} L_{1}(x) h_{1}}}{e^{-\operatorname{Pr} N_{b} L_{1}(x) h_{2}}-e^{-\operatorname{Pr} N_{b} L_{1}(x) h_{1}}}, \\
\Phi(x, y) & =\left(1+\frac{N_{t}}{N_{b}}\right)\left(\frac{y-h_{1}}{h_{2}-h_{1}}\right) \\
-\frac{N_{t}}{N_{b}} & \left(\frac{e^{-\operatorname{Pr} N_{b} L_{1}(x) y}-e^{-\operatorname{Pr} N_{b} L_{1}(x) h_{1}}}{e^{-\operatorname{Pr} N_{b} L_{1}(x) h_{2}}-e^{-\operatorname{Pr} N_{b} L_{1}(x) h_{1}}}\right) .
\end{aligned}
$$

Since the exact solutions of Eqs. (26) and (27) look difficult as they are highly non-linear differential equations, we apply the regular perturbation technique. Now, we expand $\Psi, p$ and $F$ as:

$$
\begin{aligned}
& \Psi=\Psi_{0}+\Gamma\left(\Psi_{1}\right), \\
& p=p_{0}+\Gamma\left(p_{1}\right), \\
& F=F_{0}+\Gamma\left(F_{1}\right) .
\end{aligned}
$$

Substituting Eqs. (37) to (39) into Eqs. (26) to (28), and collecting the like powers of $\Gamma$, we obtain the following systems.

\subsection{System of order $\Gamma^{0}$}

$$
\begin{aligned}
\frac{\partial^{4} \Psi_{0}}{\partial y^{4}}- & M^{2} \cos ^{2} \Theta\left(\frac{\partial^{2} \Psi_{0}}{\partial y^{2}}\right) \\
= & -\left(\operatorname{Gr} \frac{\partial \theta}{\partial y}+\operatorname{Br} \frac{\partial \Phi}{\partial y}\right), \\
\frac{\partial p_{0}}{\partial x}= & \frac{\partial^{3} \Psi_{0}}{\partial y^{3}}-M^{2} \cos ^{2} \Theta\left(\Psi_{0 y}+1\right) \\
& +\operatorname{Gr} \theta+\operatorname{Br} \Phi, \\
\Psi_{0}= & \frac{F_{0}}{2}, \quad \frac{\partial \Psi_{0}}{\partial y}=-1 \quad \text { on } y=h_{1}(x), \\
\Psi_{0}= & -\frac{F_{0}}{2}, \quad \frac{\partial \Psi_{0}}{\partial y}=-1 \quad \text { on } y=h_{2}(x) .
\end{aligned}
$$

\subsection{System of order $\Gamma^{1}$}

$$
\begin{gathered}
\frac{\partial^{4} \Psi_{1}}{\partial y^{4}}-M^{2} \cos ^{2} \Theta\left(\frac{\partial^{2} \Psi_{1}}{\partial y^{2}}\right) \\
=-2 \frac{\partial^{2}}{\partial y^{2}}\left(\frac{\partial^{2} \Psi_{0}}{\partial y^{2}}\right)^{3}, \\
\frac{\partial p_{1}}{\partial x}=\frac{\partial^{3} \Psi_{1}}{\partial y^{3}}-M^{2} \cos ^{2} \Theta \frac{\partial \Psi_{1}}{\partial y} \\
+2 \frac{\partial}{\partial y}\left(\frac{\partial^{2} \Psi_{0}}{\partial y^{2}}\right)^{3},
\end{gathered}
$$




$$
\begin{aligned}
& \Psi_{1}=\frac{F_{1}}{2}, \quad \frac{\partial \Psi_{1}}{\partial y}=0 \quad \text { on } \quad y=h_{1}(x), \\
& \Psi_{1}=-\frac{F_{1}}{2}, \quad \frac{\partial \Psi_{1}}{\partial y}=0 \quad \text { on } \quad y=h_{2}(x) .
\end{aligned}
$$

\subsection{Solution for system of order $\Gamma^{0}$}

The solution of Eq. (40), satisfying the boundary conditions, is defined as follows:

$$
\begin{gathered}
\Psi_{0}=C_{0}+C_{1} y+C_{2} \cosh \left(L_{0} y\right)+C_{3} \sinh \left(L_{0} y\right) \\
+\frac{L_{6} e^{-L_{1} \operatorname{Pr} y N_{b}}}{\left(L_{0}^{2} L_{1}^{2} \operatorname{Pr}^{2} N_{b}^{2}-L_{1}^{4} \operatorname{Pr}^{4} N_{b}^{4}\right)}+\frac{L_{5} y^{2}}{2 L_{0}^{2}},
\end{gathered}
$$

where $L_{0}=M^{2} \cos ^{2} \Theta$.

The axial pressure gradient at this order is defined as:

$$
\begin{aligned}
\frac{d p_{0}}{d x}= & \frac{L_{6} e^{-L_{1} N_{b} \operatorname{Pr} y}}{L_{1} \operatorname{Pr} N_{b}}-\left(C_{1}+1\right) L_{0}^{2}-L_{5} y \\
& +\operatorname{Gr}\left(\frac{e^{-\operatorname{Pr} N_{b} L_{1}(x) y}-e^{-\operatorname{Pr} N_{b} L_{1}(x) h_{1}}}{e^{-\operatorname{Pr} N_{b} L_{1}(x) h_{2}}-e^{-\operatorname{Pr} N_{b} L_{1}(x) h_{1}}}\right) \\
& +\operatorname{Br}\left(\left(1+\frac{N_{t}}{N_{b}}\right)\left(\frac{y-h_{1}}{h_{2}-h_{1}}\right)\right. \\
& \left.-\frac{N_{t}}{N_{b}}\left(\frac{e^{-\operatorname{Pr} N_{b} L_{1}(x) y}-e^{-\operatorname{Pr} N_{b} L_{1}(x) h_{1}}}{e^{-\operatorname{Pr} N_{b} L_{1}(x) h_{2}}-e^{-\operatorname{Pr} N_{b} L_{1}(x) h_{1}}}\right)\right) .
\end{aligned}
$$

For one wavelength, the integration of Eq. (49) yields:

$$
\Delta p_{0}=\int_{0}^{1}\left(\frac{d p_{0}}{d x}\right)_{y=o} d x
$$

where $C_{0}, C_{1}, C_{2}$ and $C_{3}$ are constants. These constants are calculated with the help of boundary conditions. The remaining constants $L_{i}^{\prime} s, i=5$ to 45 are used to simplify the equations. All these constants are defined in the Appendix.

\subsection{Solution for system of order $\Gamma^{1}$}

After substituting zeroth-order solution (48) into Eq. (44), the solution, satisfying the boundary conditions, is defined as follows

$$
\begin{aligned}
\Psi_{1}= & C_{4}+C_{5} y+C_{6} \cosh \left(L_{0} y\right)+C_{7} \sinh \left(L_{0} y\right) \\
& +L_{28} e^{-3 L_{1} \operatorname{Pr} y N_{b}}+L_{29} e^{-2 L_{1} \operatorname{Pr} y N_{b}} \frac{1}{4}\left(L_{24} e^{4 L_{0} y}\right. \\
& +L_{25} e^{3 L_{0} y}+L_{26} e^{L_{0} y}+L_{27} e^{2 L_{0} y} \\
& \left.+L_{23}\right) e^{-y\left(L_{1} \operatorname{Pr} N_{b}+2 L_{0}\right)}+L_{34} y \sinh \left(L_{0} y\right) \\
& +L_{30} e^{2 L_{0} y-y\left(2 L_{1} \operatorname{Pr} N_{b}+L_{0}\right)}
\end{aligned}
$$

$$
\begin{aligned}
& +L_{31} e^{-y\left(2 L_{1} \operatorname{Pr} N_{b}+L_{0}\right)}+L_{35} \sinh \left(L_{0} y\right) \\
& +L_{36} \sinh \left(3 L_{0} y\right)+L_{32} \cosh \left(3 L_{0} y\right) \\
& +L_{33} \cosh \left(L_{0} y\right)+L_{37} y \cosh \left(L_{0} y\right) \\
& +\frac{L_{11} \sinh \left(2 L_{0} y\right)}{L_{0}^{4}}+\frac{L_{12} \cosh \left(2 L_{0} y\right)}{12 L_{0}^{4}} .
\end{aligned}
$$

The axial pressure gradient at this order is defined as:

$$
\begin{aligned}
& \frac{d p_{1}}{d x}=+\frac{1}{4}\left(\left(96\left(\sinh \left(3 y L_{0}\right) L_{32}+\cosh \left(3 y L_{0}\right) L_{36}\right)\right.\right. \\
& \left.-6 e^{-y\left(2 L_{0}+\operatorname{Pr} L_{1} N_{b}\right)}\left(L_{23}-e^{4 y L_{0}} L_{24}\right)\right) L_{0}^{3} \\
& -4 C_{5} L_{0}^{2}+\left(8 \sinh \left(y L_{0}\right) L_{34}+8 \cosh \left(y L_{0}\right) L_{37}\right. \\
& +\operatorname{Pr} L_{1}\left(-11 e^{-y\left(2 L_{0}+\operatorname{Pr} L_{1} N_{b}\right)} L_{23}\right. \\
& -11 e^{2 y L_{0}-\operatorname{Pr} y L_{1} N_{b}} L_{24}-2 e^{y\left(L_{0}-\operatorname{Pr} L_{1} N_{b}\right)} L_{25} \\
& -2 e^{-y\left(L_{0}+\operatorname{Pr} L_{1} N_{b}\right)} L_{26}+e^{-\operatorname{Pr} y L_{1} N_{b}} L_{27} \\
& +12 e^{-3 \operatorname{Pr} y L_{1} N_{b}} L_{28}+8 e^{-2 \operatorname{Pr} y L_{1} N_{b}} L_{29} \\
& -16 e^{y\left(L_{0}-2 \operatorname{Pr} L_{1} N_{b}\right)} L_{30} \\
& \left.\left.-16 e^{-y\left(L_{0}+2 \operatorname{Pr} L_{1} N_{b}\right)} L_{31}\right) N_{b}\right) L_{0}^{2} \\
& -3 e^{-2 y\left(L_{0}+\operatorname{Pr} L_{1} N_{b}\right)}(\operatorname{Pr})^{2} L_{1}^{2}\left(2 e^{\operatorname{Pr} y L_{1} N_{b}} L_{23}\right. \\
& +e^{y L_{0}}\left(-2 e^{3 y L_{0}+\operatorname{Pr} y L_{1} N_{b}} L_{24}\right. \\
& -e^{y\left(2 L_{0}+\operatorname{Pr} L_{1} N_{b}\right)} L_{25}+e^{\operatorname{Pr} y L_{1} N_{b}} L_{26} \\
& \left.\left.-16 e^{2 y L_{0}} L_{30}+16 L_{31}\right)\right) N_{b}^{2} L_{0} \\
& +(\operatorname{Pr})^{3} L_{1}^{3}\left(-e^{-y\left(2 L_{0}+\operatorname{Pr} L_{1} N_{b}\right)} L_{23}\right. \\
& -e^{2 y L_{0}-\operatorname{Pr} y L_{1} N_{b}} L_{24}-e^{y\left(L_{0}-\operatorname{Pr} L_{1} N_{b}\right)} L_{25} \\
& -e^{-y\left(L_{0}+\operatorname{Pr} L_{1} N_{b}\right)} L_{26}-e^{-\operatorname{Pr} y L_{1} N_{b}} L_{27} \\
& -108 e^{-3 \operatorname{Pr} y L_{1} N_{b}} L_{28}-32 e^{-2 \operatorname{Pr} y L_{1} N_{b}} L_{29} \\
& -32 e^{y\left(L_{0}-2 \operatorname{Pr} L_{1} N_{b}\right)} L_{30} \\
& \left.-32 e^{-y\left(L_{0}+2 \operatorname{Pr} L_{1} N_{b}\right)} L_{31}\right)\left(N_{b}\right)^{3} \\
& \left.+\frac{2\left(12 \cosh \left(2 y L_{0}\right) L_{11}+\sinh \left(2 y L_{0}\right) L_{12}\right)}{L_{0}}\right) \\
& +6\left(\sinh \left(y L_{0}\right) C_{2} L_{0}^{3}+\cosh \left(y L_{0}\right) C_{3} L_{0}^{3}\right.
\end{aligned}
$$




$$
\begin{aligned}
& \left.-\frac{e^{-\operatorname{Pr} y L_{1} N_{b}} \operatorname{Pr}^{3} L_{1}^{3} L_{6}\left(N_{b}\right)^{3}}{\operatorname{Pr}^{2} L_{0}^{2} L_{1}^{2} N_{b}^{2}-\operatorname{Pr}^{4} L_{1}^{4} N_{b}^{4}}\right)\left(\cosh \left(y L_{0}\right) C_{2} L_{0}^{2}\right. \\
& +\sinh \left(y L_{0}\right) C_{3} L_{0}^{2}+\frac{L_{5}}{L_{0}^{2}} \\
& \left.+\frac{e^{-\operatorname{Pr} y L_{1} N_{b}} \operatorname{Pr}^{2} L_{1}^{2} L_{6} N_{b}^{2}}{\operatorname{Pr}^{2} L_{0}^{2} L_{1}^{2} N_{b}^{2}-\operatorname{Pr}^{4} L_{1}^{4} N_{b}^{4}}\right)^{2}
\end{aligned}
$$

For one wavelength, the integration of Eq. (52) yields:

$$
\Delta p_{1}=\int_{0}^{1}\left(\frac{d p_{1}}{d x}\right)_{y=o} d x
$$

where $C_{4}, C_{5}, C_{6}$ and $C_{7}$ are constants. These constants are calculated with the help of boundary conditions. The remaining constants $L_{i}^{\prime} s, i=5$ to 45 are used to simplify the equations. All these constants are defined in the Appendix.

Summarizing the perturbation results for small parameter $\Gamma$, we have:

$$
\begin{aligned}
& \Psi=\Psi_{0}+\Gamma \Psi_{1}, \\
& \frac{d p}{d x}=\frac{d p_{0}}{d x}+\Gamma \frac{d p_{1}}{d x}, \\
& \Delta p=\Delta p_{0}+\Gamma \Delta p_{1},
\end{aligned}
$$

defining:

$$
F=F_{0}+\Gamma F_{1},
$$

and substituting:

$$
F_{0}=F-\Gamma F_{1},
$$

in Eqs. (54) to (56) results will only be in terms of $O(\Gamma)$.

\section{Numerical results and discussion}

This section deals with the graphical results of the existing flow problem. Mathematica software is used to compute the numerical solutions of pressure rise and pressure gradient. Figures 1-14 are displayed for different wave forms in order to see the effects of various parameters on various quantities such as pressure rise, pressure gradient, stream lines, temperature, and concentration distribution. Figures 1 to 4 describe the pumping phenomena for different values of Gr, $M, \Gamma$, and $N_{t}$. It is observed from Figure 1 that in the retrograde pumping $(\Delta p>0, Q<0)$, peristaltic $(\Delta p>0, Q>0)$, and augmented pumping $(\Delta p<0, Q>0)$ regions, the pressure rise increases with an increase in Gr. It is depicted in Figure 2 that with an increase in $M$, the pressure rise increases

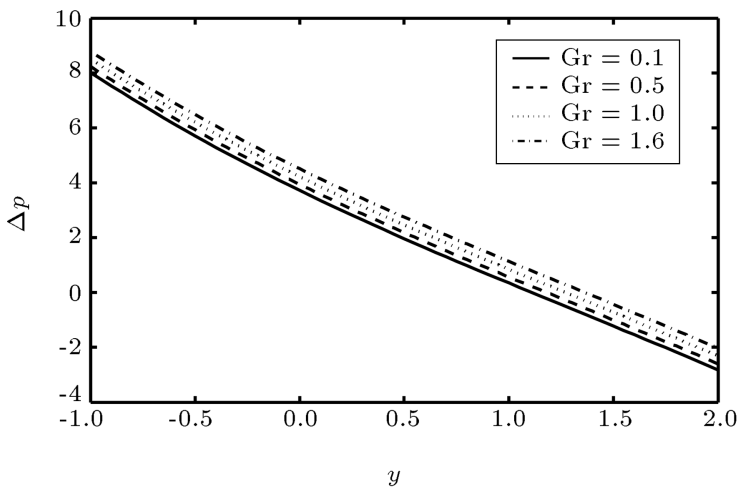

Figure 1. Variation of pressure rise $\Delta p$ for different values of $\mathrm{Gr}$. The other parameters are $a=0.7, b=0.9$, $\mathrm{Br}=0.8, \Gamma=0.01, d=1.5, \Theta=\frac{\pi}{7}, M=1, \operatorname{Pr}=0.7$, $\phi=0.8, N_{b}=0.9$ and $N_{t}=0.1$.

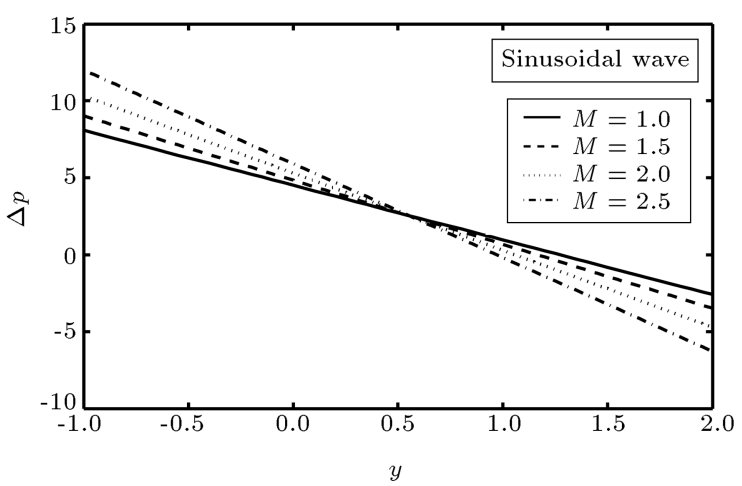

Figure 2. Variation of pressure rise $\Delta p$ for different values of $M$. The other parameters are $a=0.7, b=0.9$, $\mathrm{Br}=0.8, \Gamma=0.0001, d=1.5, \mathrm{Gr}=0.8, \Theta=\frac{\pi}{7}, \operatorname{Pr}=0.5$, $\phi=\frac{\pi}{6}, N_{b}=0.8$ and $N_{t}=0.5$.

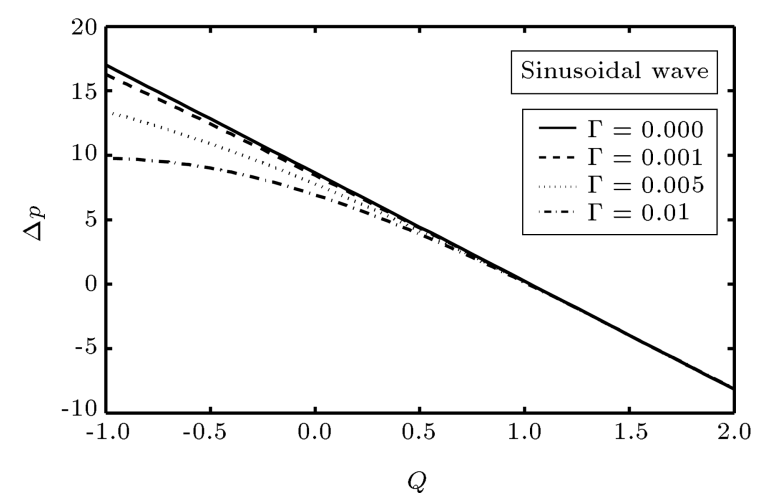

Figure 3. Variation of pressure rise $\Delta p$ for different values of $\Gamma$. The other parameters are $a=0.7, b=1$, $\mathrm{Br}=0.8, d=1.5, \mathrm{Gr}=0.8, \Theta=\frac{\pi}{7}, M=3, \operatorname{Pr}=0.5$, $\phi=\frac{\pi}{6}, N_{b}=0.9$ and $N_{t}=0.5$.

in the retrograde pumping $(\Delta p>0, Q<0)$ and peristaltic $(\Delta p>0, Q>0)$ pumping regions, while in augmented pumping $(\Delta p<0, Q>0)$ region, the behavior is quite opposite. Here, the pressure rise decreases with an increase in $M$. Figure 3 shows the variations of pressure rise for different values of $\Gamma$. It is 


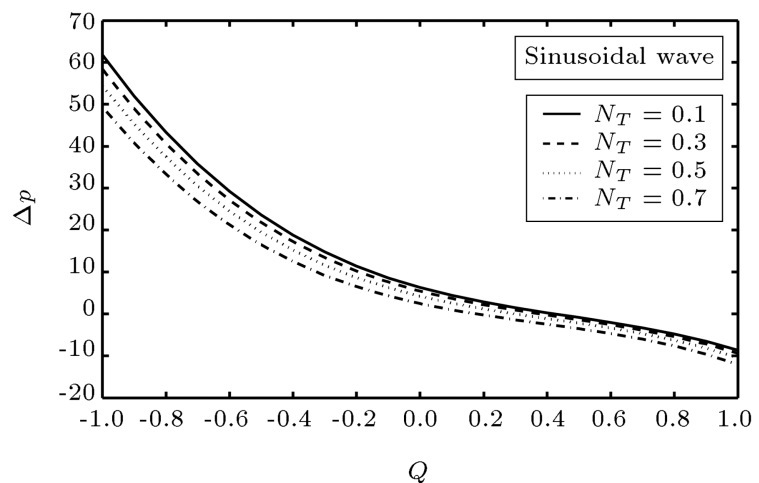

Figure 4. Variation of pressure rise $\Delta p$ for different values of $N_{t}$. The other parameters are $a=0.7, b=1$, $\mathrm{Br}=0.8, d=1.5, \mathrm{Gr}=0.8, \Theta=\frac{\pi}{7}, M=3, \operatorname{Pr}=0.5$, $\phi=\frac{\pi}{6}, N_{b}=0.9$ and $\Gamma=0.001$.

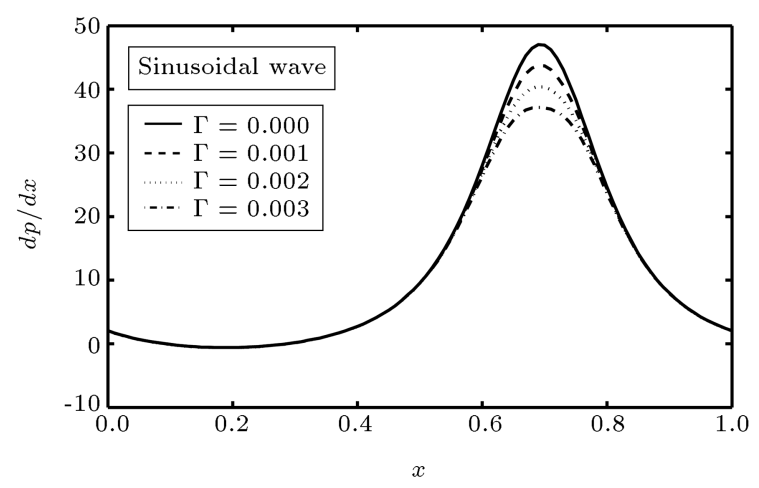

Figure 5. Variation of pressure gradient $d p / d x$ for different values of $\Gamma$. The other parameters are $a=0.5$, $b=1, \mathrm{Br}=0.8, d=1.5, \mathrm{Gr}=0.8, \Theta=\frac{\pi}{7}, M=3$, $\operatorname{Pr}=0.8, N_{b}=0.9, N_{t}=0.5, Q=-1$ and $\phi=\frac{\pi}{6}$.

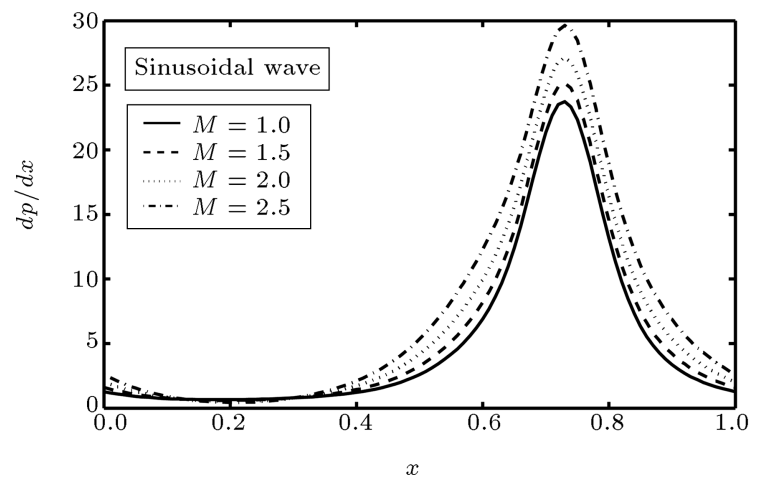

Figure 6. Variation of pressure gradient $d p / d x$ for different values of $M$. The other parameters are $a=0.7$, $b=0.5, \mathrm{Br}=0.8, \Gamma=0.01, d=1.5, \mathrm{Gr}=0.8, \Theta=\frac{\pi}{7}$, $\operatorname{Pr}=0.9, \phi=\frac{\pi}{6}, N_{t}=0.2, Q=-1$ and $N_{b}=0.8$.

observed that in the retrograde and peristaltic pumping regions, the pressure rise decreases with an increase in $\Gamma$, but there is variation of pressure rise in case of augmented pumping region. Figure 4 shows the pumping phenomena for different values of $N_{t}$. It is observed that with an increase in $N_{t}$, the pressure rise decreases in all the pumping regions. Figures 5 to 7 are plotted to see the behavior of pressure gradient for different values of $\Gamma, M$, and $Q$. It is observed form Figures 5 and 6 that the pressure gradient decreases with an increase in the value of $\Gamma$ and increases with an increase in the value of $M$. Figure 7 shows the pressure gradient for different wave forms. It is observed that the pressure gradient is maximum for the square and trapezoidal waves. In order to see the behavior of temperature and concentration profiles, Figures 8-11 are plotted. Figures 8 and 9 show the temperature profile for different values of $N_{t}$ and $\operatorname{Pr}$ by considering different wave forms. It is observed that the temperature profile increases with an increase in the values of $N_{t}$ and $\mathrm{Pr}$. Moreover, it is also observed that the temperature is maximum for the sinusoidal and trapezoidal waves. Concentration profile for different values of $N_{t}$ and $N_{b}$ is shown in Figures 10 and 11. It is depicted that the concentration profile gives opposite behavior as compared to the temperature profile. This behavior is physically valid, because concentration shows an inverse relationship with temperature. Here, it is also observed that the concentration profile decreases with an increase in $N_{t}$ and increases with an increase in $N_{b}$. The concentration profile is maximum for the sinusoidal and trapezoidal waves.

Stream lines for different values of $M$ and $\mathrm{Br}$ are shown in Figures 12 and 13. It is depicted in Figure 12 that the size of the trapping bolus decreases with an increase in $M$. It is observed form Figure 13 that with an increase in $\mathrm{Br}$, the size of trapping bolus decreases in the lower half of the channel, while in the upper half of the channel, the size of the trapping bolus increases. Figure 14 shows the stream lines for different wave forms.

\section{Concluding remarks}

This research paper considered the nanofluid effects on peristaltic transport of a fourth grade fluid in the occurrence of inclined magnetic field. The main findings are summarized as follows:

1. Pressure rise increases with an increase in $\mathrm{Gr}$ in the retrograde, peristaltic, and augmented pumping regions;

2. With an increase in $M$, the pressure rise increases in the retrograde and peristaltic pumping regions, while in augmented pumping region, the behavior is quite opposite;

3. Pressure rise decreases in the retrograde and peristaltic pumping regions with an increase in the value of $\Gamma$;

4. Pressure rise decreases in all the pumping regions with an increase in $N_{t}$; 


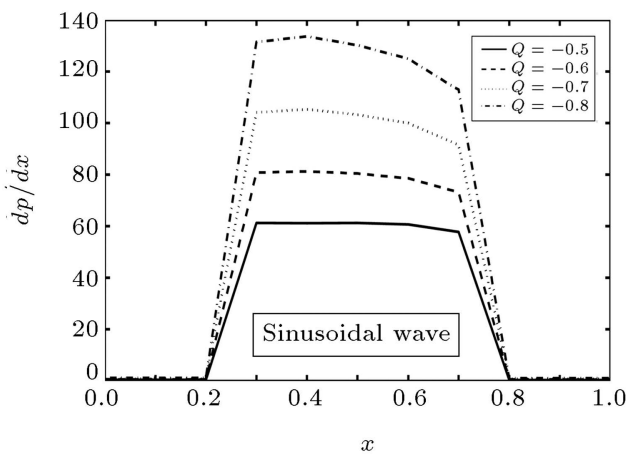

(a)

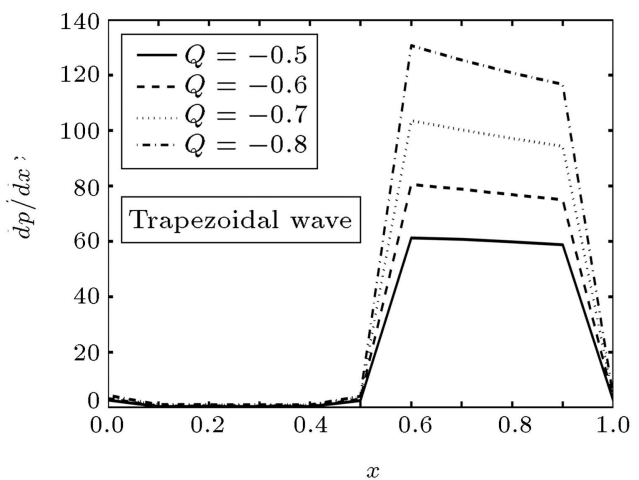

(c)

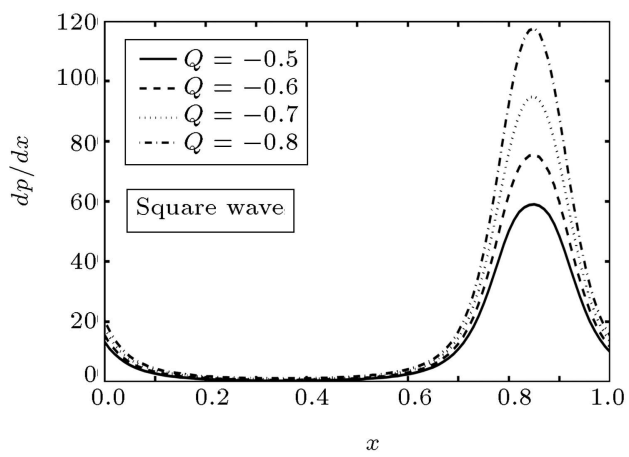

(b)

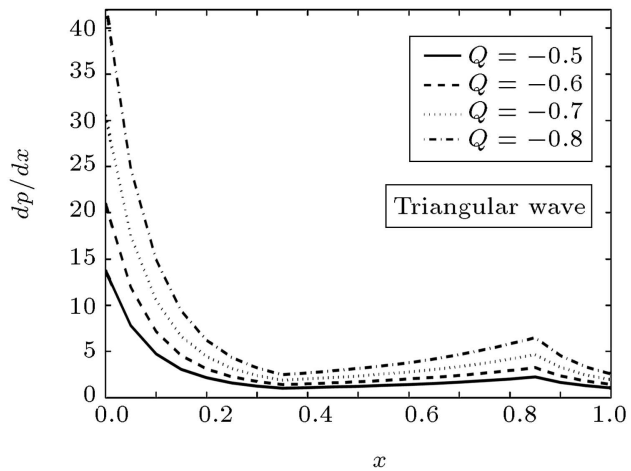

(d)

Figure 7. Variation of pressure gradient $d p / d x$ for different wave form for (a) square wave, (b) sinusoidal wave, (c) trapezoidal wave, and (d) triangular wave. The other parameters are $a=0.3, b=0.7, \mathrm{Br}=0.8, \Gamma=0.05, d=1.5$, $\mathrm{Gr}=0.8, \Theta=\frac{\pi}{8}, \operatorname{Pr}=0.9, \phi=0.1, N_{t}=0.8, Q=-3$ and $N_{b}=0.5$.

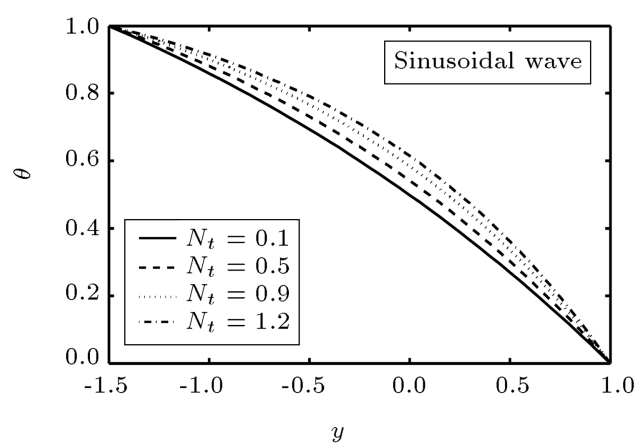

(a)

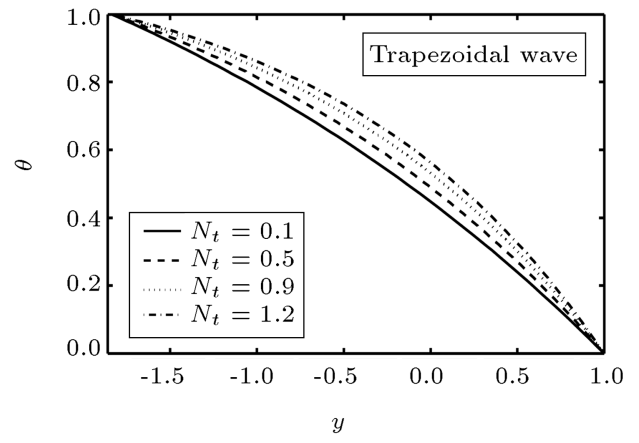

(c)

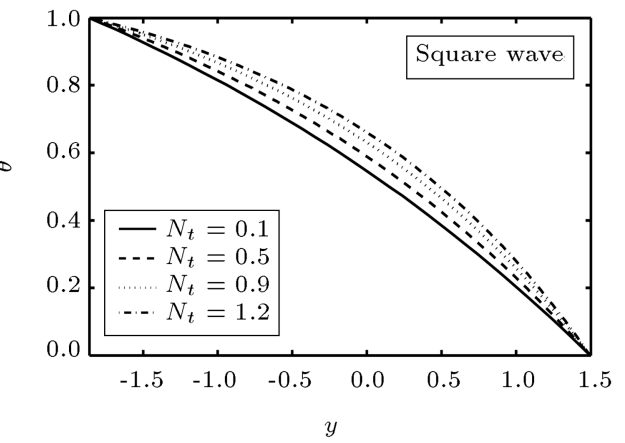

(b)

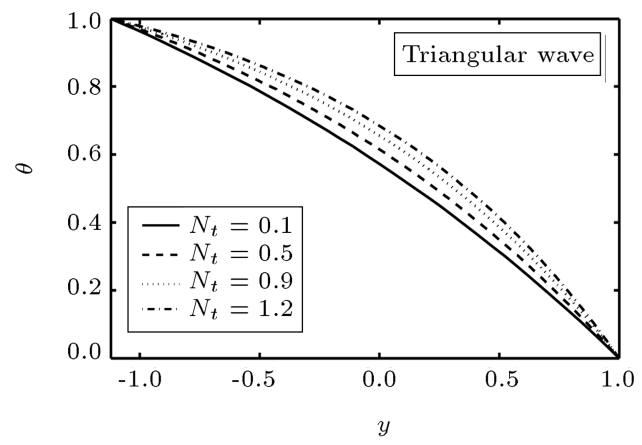

(d)

Figure 8. Temperature profile for different wave forms for (a) sinusoidal wave, (b) square wave, (c) trapezoidal wave, and (d) triangular wave. The other parameters are $a=0.5, b=1, N_{b}=0.8, d=1, \operatorname{Pr}=0.9, x=0$ and $\phi=\frac{\pi}{6}$. 


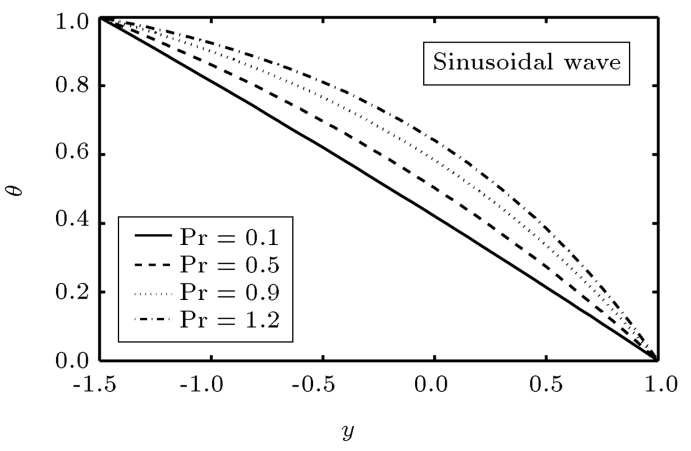

(a)

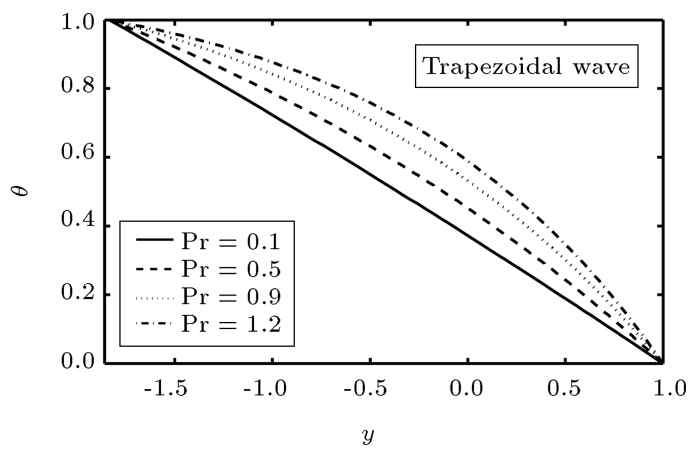

(c)

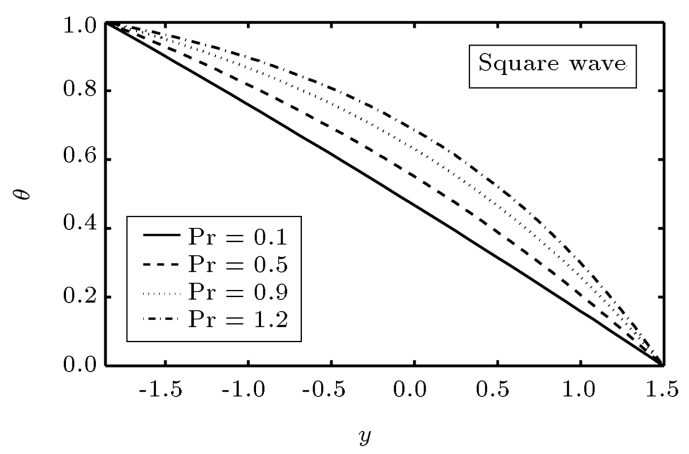

(b)

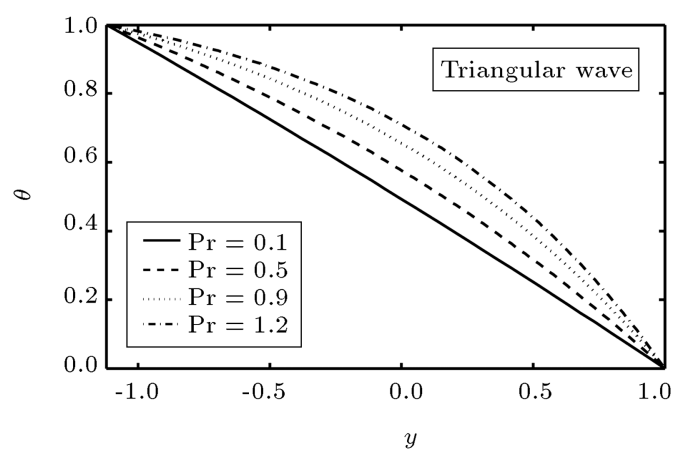

(d)

Figure 9. Temperature profile for different wave forms for (a) sinusoidal wave, (b) square wave, (c) trapezoidal wave, and (d) triangular wave. The other parameters are $a=0.5, b=1, N_{b}=0.8, d=1, x=0, \phi=\frac{\pi}{6}$ and $N_{t}=0.9$.

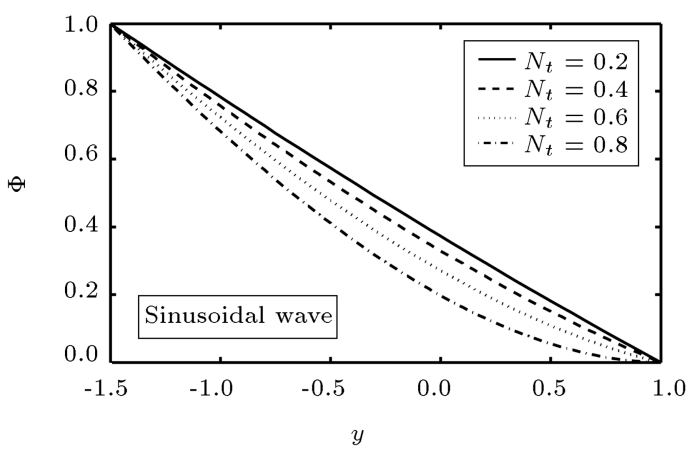

(a)

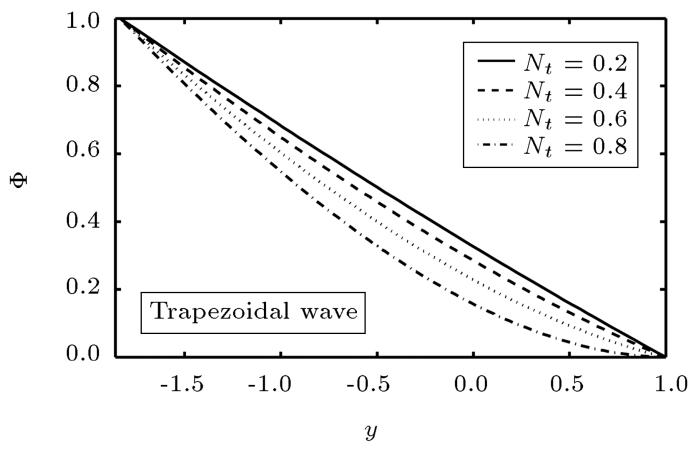

(c)

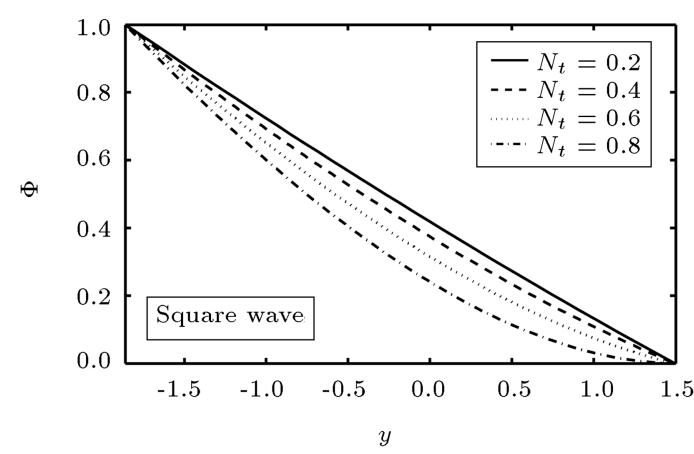

(b)

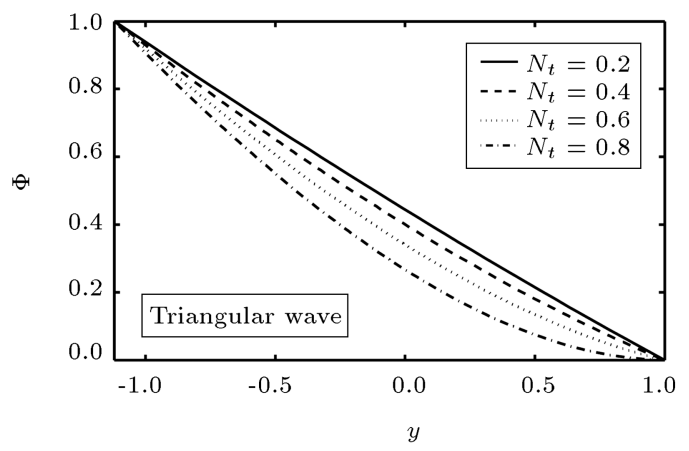

(d)

Figure 10. Concentration profile for different wave forms for (a) sinusoidal wave, (b) square wave, (c) trapezoidal wave, and (d) triangular wave. The other parameters are $a=0.5, b=1, N_{b}=0.5, d=1, \operatorname{Pr}=0.8, x=0$ and $\phi=\frac{\pi}{6}$. 


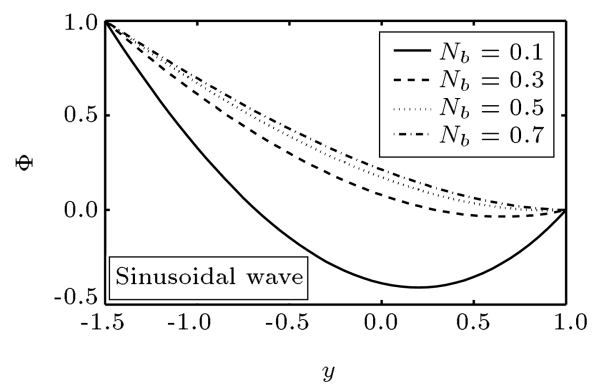

(a)

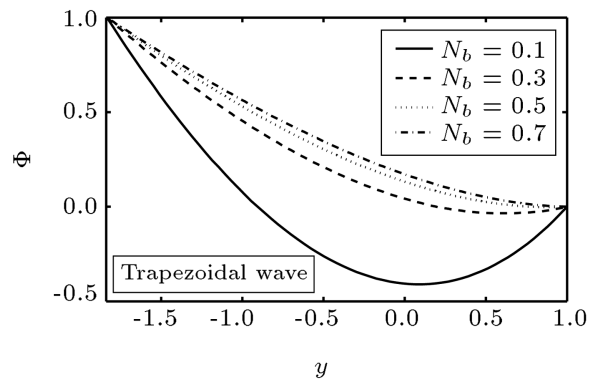

(c)

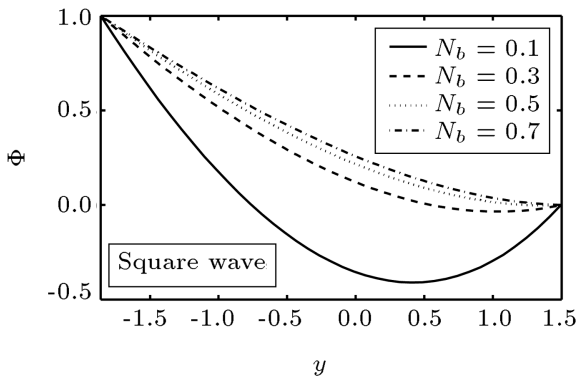

(b)

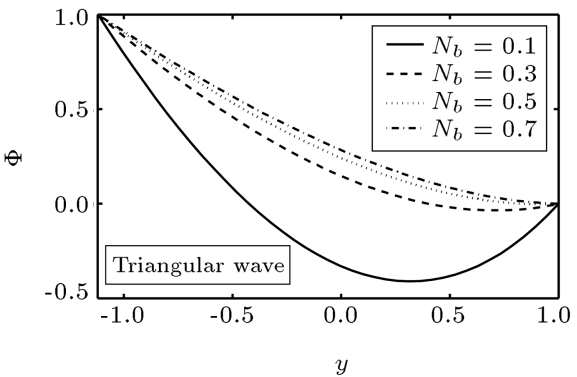

(d)

Figure 11. Concentration profile for different wave forms for (a) sinusoidal wave, (b) square wave, (c) trapezoidal wave, and (d) triangular wave. The other parameters are $a=0.5, b=1, \operatorname{Pr}=0.9, d=1, \phi=\frac{\pi}{6}$ and $N_{t}=0.8$.

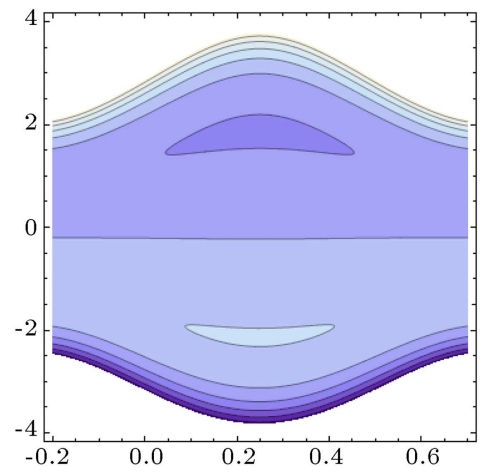

(a)

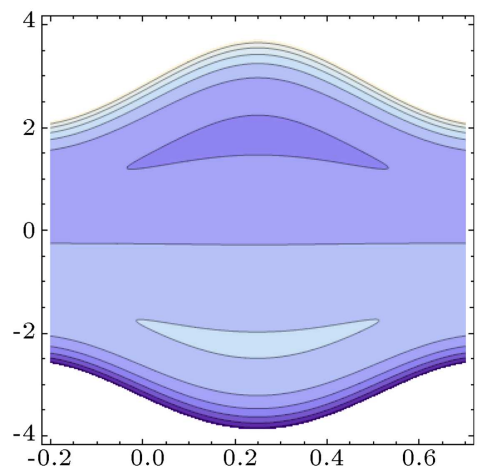

(b)

Figure 12. Stream lines for different values of $M$ for sinusoidal wave for (a) $M=1.5$, and (b) $M=1.6$. The other parameters are $a=0.3, b=0.3, \mathrm{Br}=0.8, d=1.4, \mathrm{Gr}=0.8, \operatorname{Pr}=0.3, \phi=0.01, N_{b}=0.7, \Gamma=0.001, \Theta=\frac{\pi}{8}, Q=-2.2$ and $N_{t}=0.3$.

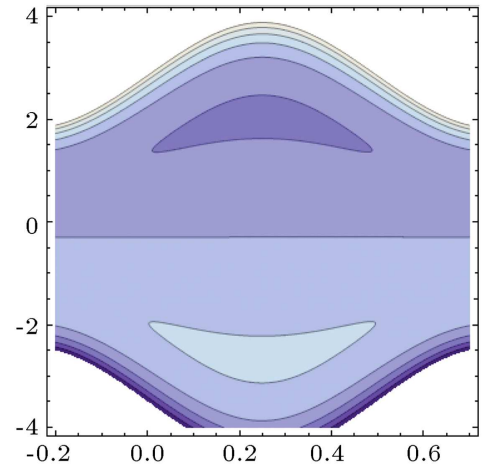

(a)

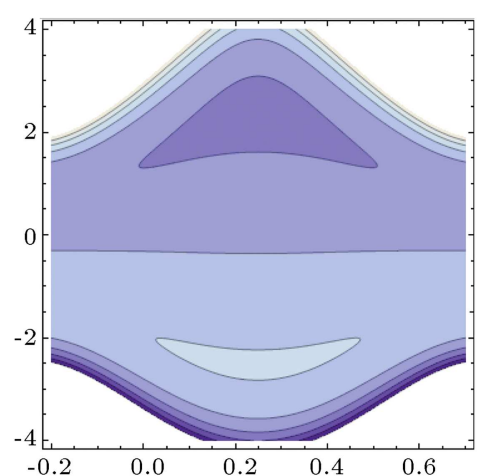

(b)

Figure 13. Stream lines for different values of $\mathrm{Br}$ for sinusoidal wave for (a) $\mathrm{Br}=0.1$, and (b) $\mathrm{Br}=0.5$. The other parameters are $a=0.4, b=0.4, d=1.6, \mathrm{Gr}=0.5, M=1.5, \operatorname{Pr}=0.1, \phi=0.01, N_{b}=0.5, \Gamma=0.002, \Theta=\frac{\pi}{8}, Q=-2$ and $N_{t}=0.5$. 


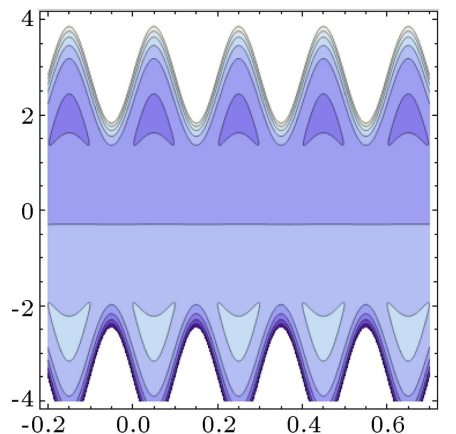

(a)

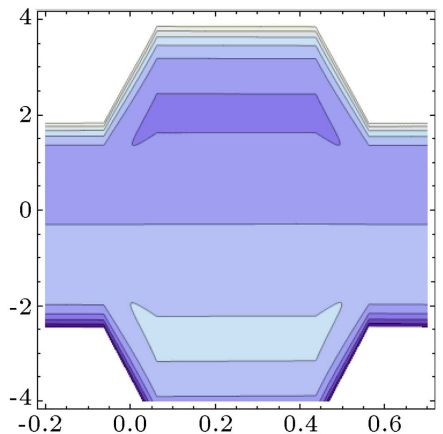

(b)

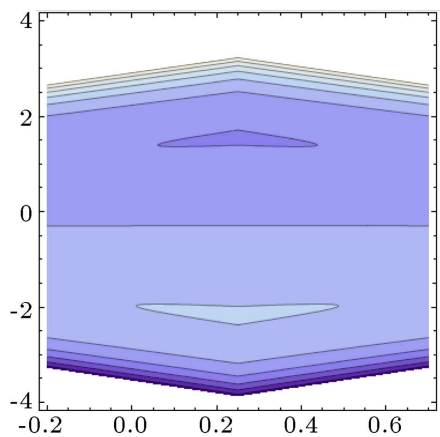

(c)

Figure 14. Stream lines for different wave forms for (a) trapezoidal wave, (b) square wave, and (c) triangular wave. The other parameters are $a=0.4, b=0.4, \mathrm{Br}=0.1, d=1.6, \mathrm{Gr}=0.5, M=1.5, \operatorname{Pr}=0.1, \phi=0.01, N_{b}=0.9, \Gamma=0.002$, $\Theta=\frac{\pi}{8}, Q=-2$ and $N_{t}=0.5$.

5. Pressure gradient decreases with an increase in the value of $\Gamma$ and increases with an increase in the value of $M$;

6. Temperature profile increases with increase in the values of $N_{t}$ and $\mathrm{Pr}$

7. Concentration profile decreases with an increase in $N_{t}$ and increases with an increase in $N_{b}$;

8. The size of trapping bolus decreases with an increase in $M$;

9. With an increase in $\mathrm{Br}$, the size of trapping bolus decreases in the lower half of the channel, while in the upper half of the channel, the size of trapping bolus increases.

\section{References}

1. Eldabe, N.T., El-Sayed, M.F., Ghaly, A.Y. and Sayed, H.M. "Peristaltically induced transport of a MHD biviscosity fluid in a non-uniform tube", Physica A, 383(2), pp. 252-266 (2007).

2. Nadeem, S., Akbar, N.S. and Hayat, T. "Effects of variable viscosity on the peristaltic motion in a thirdorder fluid", Z. Naturforsch. A, 65a, pp. 901-910 (2010).

3. Akram, S., Nadeem, S. and Hussain, A. "Influence of induced magnetic field and partial slip on the peristaltic flow of a couple stress fluid in an asymmetric channel", Iran. J. Chem. Chem. Eng., 33(3), pp. 43-52 (2014).

4. Akram, S. and Nadeem, S. "Analytical analysis of peristaltic flow of a six constant Jeffrey's model of fluid in an inclined planar channel", Walailak J. of Sci. and Technology, 11(2), pp. 129-148 (2014).

5. Ellahi, R., Riaz, A., Sohail, S. and Mushtaq, M. "Series solutions of magnetohydrodynamic peristaltic flow of a Jeffrey fluid in eccentric cylinders", Appl. Maths and Inform. Sci., 7(4), pp. 1441-1449 (2013).
6. Akram, S., Nadeem, S. and Hanif, M. "Numerical and analytical treatment on peristaltic flow of Williamson fluid in the occurrence of induced magnetic field", $J$. Magn. Magn. Mater., 346, pp. 142-151 (2013).

7. Akram, S., Mekheimer, Kh.S. and Nadeem, S. "Influence of lateral walls on peristaltic flow of a couple stress fluid in a non-uniform rectangular duct", Appl. Maths and Inform. Sci., 8(3), pp. 1127-1133 (2014).

8. Nadeem, S., Akram, S. and Akbar, N.S. "Simulation of heat and chemical reactions on peristaltic flow of a Williamson fluid in an inclined asymmetric channel", Iran. J. Chem. Chem. Eng., 32(2), pp. 93-107 (2013).

9. Latham, T.W., Fluid Motion in Peristaltic Pump, MS thesis, MIII, Cambridge, Mass (1966).

10. Shapiro, A.H., Jaffrin, M.Y. and Weinberg, S.L. "Peristaltic pumping with long wavelengths at low Reynolds number", J. of Fluid Mech., 37(4), pp. 799-825 (1969).

11. Akram, S. and Nadeem, S. "Influence of induced magnetic field and heat transfer on the peristaltic motion of a Jeffrey fluid in an asymmetric channel: Closed form solutions", J. Magn. Magn. Mater., 328, pp. 11-20 (2013).

12. Mekheimer, Kh.S. "Peristaltic flow of blood under effect of a magnetic field in a non-uniform channels", Appl. Math. Comput., 153(3), pp. 763-777 (2004).

13. Mekheimer, Kh.S. and Al-Arabi, T.H. "Nonlinear peristaltic transport of MHD flow through a porous medium", Inter. J. of Math. and Mathematical Sci., 2003(26), pp. 1663-1682 (2003).

14. Elshehawey, E.F., Elsayed Elbarbary, M.E. and Nasser Elgazery, S. "Effects of inclined magnetic field on magneto fluid flow through a porous medium between two inclined wavy porous plates (numerical study)", Appl. Math. Comput., 135(1), pp. 85-103 (2003).

15. Mekheimer, Kh.S. and Abd Elmaboud, Y. "The influence of heat transfer and magnetic field on peristaltic transport of a Newtonian fluid in a vertical annulus: 
An application of an endoscope", Phy. Letters A., 372(10), pp. 1657-1665 (2008).

16. Nadeem, S. and Akram, S. "Magnetohydrodynamic peristaltic flow of a hyperbolic tangent fluid in a vertical asymmetric channel with heat transfer", Acta Mech. Sin., 27(2), pp. 237-250 (2011).

17. Akram, A., Nadeem, S. and Hussain, A. "Effects of heat and mass transfer on peristaltic flow of a Bingham fluid in the presence of inclined magnetic field and channel with different wave forms", J. Magn. Magn. Mater., 362, pp. 184-192 (2014).

18. Kothandapani, M. and Srinivas, S. "Peristaltic transport of a Jeffrey fluid under the effect of magnetic field in an asymmetric channel", Inter. J. of Non-Linear Mech., 43(9), pp. 915-924 (2008).

19. Minkowycz, W.J., Sparrow, E.M. and Abraham, J.P., Nanoparticle Heat Transfer and Fluid Flow, CRC Press, Taylor \& Francis (2013).

20. Choi, S.U.S. "Enhancing thermal conductivity of fluid with nanoparticles developments and applications of non-Newtonian flow", ASME FED, 66, pp. 99-105 (1995).

21. Khan, W.A. and Pop, I. "Boundary-layer flow of a nanofluid past a stretching sheet", Inter. J. of Heat Mass Transfer, 53(11-12), pp. 2477-2483 (2010).

22. Khanafer, K., Vafai, K. and Lightstone, M. "Buoyancy-driven heat transfer enhancement in a twodimensional enclosure utilizing nanofluids", Inter. J. of Heat Mass Transfer, 46(19), pp. 3639-3653 (2003).

23. Sheikholeslamia, M., Ganjia, D.D., Javed, M.Y. and Ellahi, R. "Effect of thermal radiation on magnetohydrodynamics nanofluid flow and heat transfer by means of two phase model", J. Magn. Magn. Mater., 374, pp. 36-43 (2015).

24. Akbar, N.S., Raza, M. and Ellahi, R. "Influence of heat generation and heat flux on peristaltic flow with interacting nanoparticles", Eur. Phys. J. Plus, 129, p. 185 (2014).

25. Sheikholeslami, M., Ellahi, R., Mohsan Hassan and Soheil Soleimani, S. "A study of natural convection heat transfer in a nanofluid filled enclosure with elliptic inner cylinder", Inter. J. of Num. Meth. for Heat \& Fluid Flow, 24(8), pp. 1906-1927 (2014).

26. Sheikholeslami, M., Ellahi, R., Ashorynejad, H.R., Domairry, G. and Hayat, T. "Effects of heat transfer in flow of nanofluids over a permeable stretching wall in a porous medium", J. of Comput. and Theoretical Nanoscience, 11(2), pp. 486-496 (2014).

27. Sheikholeslamia, M., Bandpy, M.G., Ellahi, R. and Zeeshan, A. "Simulation of MHD CuO-water nanofluid flow and convective heat transfer considering Lorentz forces", J. Magn. Magn. Mater., 369, pp. 69-80 (2014).

28. Akbar, N.S., Raza, M. and Ellahi, R. "Interaction of nano particles for the peristaltic flow in an asymmetric channel with the induced magnetic field", Eur. Phys. J. Plus., 129, pp. 155-167 (2014).

29. Akbar, N.S., Rahman, S.U., Ellahi, R. and Nadeem, S. "Nano fluid flow in tapering stenosed arteries with permeable walls", Inter. J. of Thermal Sci., 85, pp. 54-61 (2014).

30. Ellahi, R., Aziz, S. and Zeeshan, A. "Non Newtonian nanofluids flow through a porous medium between two coaxial cylinders with heat transfer and variable viscosity", J. of Porous Media, 16(3), pp. 205-216 (2013).

31. Ellahi, R. "The effects of MHD and temperature dependent viscosity on the flow of non-Newtonian nanofluid in a pipe: Analytical solutions", Appl. Math. Model., 37(3), pp. 1451-1457 (2013).

32. Zeeshan, A., Ellahi, R. and Hassan, M. "Magnetohydrodynamic flow of water/ethylene glycol based nanofluids with natural convection through porous medium", Eur. Phys. J. Plus., 129, p. 261 (2014).

33. Akram, S., Nadeem, S., Abdul Ghafoor, and Changhoon Lee, "Consequences of nanofluid on Peristaltic flow in an asymmetric channel", Inter. J. of Basic \& Appl. Sci., 12(5), pp. 75-96 (2012).

34. Akram, S. and Nadeem, S. "Significance of nanofluid and partial slip on the peristaltic transport of a Jeffrey fluid model in an asymmetric channel with different wave forms", IEEE Transactions on Nanotechnology, 13(2), pp. 375-385 (2014).

35. Akram, S. and Nadeem, S. "Consequence of nanofluid on Peristaltic transport of a hyperbolic tangent fluid model in the occurrence of apt (tending) magnetic field", J. Magn. Magn. Mater., 358-359, pp. 183-19 (2014).

36. Ellahi, R. "The thermodynamics, stability, applications and techniques of differential type: A review", Reviews in Theor. Sci., 2(2), pp. 16-123 (2014).

\section{Appendix}

The constants appeared in the manuscript are defined as follows:

$$
\begin{aligned}
& L_{5}=\frac{B h_{1} N_{t}}{\left(h_{1}-h_{2}\right) N_{b}}+\frac{B h_{1}}{h_{1}-h_{2}}, \\
& L_{6}=\frac{L_{1} \operatorname{Pr}\left(\operatorname{Br} N_{t}-\mathrm{Gr} N_{b}\right)}{e^{-h_{2} L_{1} \operatorname{Pr} N_{b}}-e^{-h_{1} L_{1} \operatorname{Pr} N_{b}}}, \\
& L_{7}=3 C_{2}\left(C_{2}^{2}+2 C_{3}^{2}\right) L_{0}^{8}, \\
& L_{8}=3 C_{2}\left(2 C_{2}^{2}+7 C_{3}^{2}\right) L_{0}^{8}, \\
& L_{9}=3 C_{3}\left(2 C_{2}^{2}+C_{3}^{2}\right) L_{0}^{8}, \\
& L_{10}=3 C_{3}\left(7 C_{2}^{2}+2 C_{3}^{2}\right) L_{0}^{8},
\end{aligned}
$$




$$
\begin{aligned}
& L_{11}=C_{2} C_{3} L_{0}^{4} L_{5}, \\
& L_{12}=6\left(C_{2}^{2}+C_{3}^{2}\right) L_{0}^{4} L_{5}, \\
& L_{13}=\frac{3 L_{0}^{2} L_{6}^{2}\left(C_{2}\left(4 L_{1}^{2} \operatorname{Pr}^{2} N_{b}^{2}+L_{0}^{2}\right)-4 C_{3} L_{0} L_{1} \operatorname{Pr} N_{b}\right)}{\left(L_{0}^{2}-L_{1}^{2} \operatorname{Pr}^{2} N_{b}^{2}\right)^{2}}, \\
& L_{14}=\frac{3 L_{0}^{2} L_{6}^{2}\left(C_{3}\left(4 L_{1}^{2} \operatorname{Pr}^{2} N_{b}^{2}+L_{0}^{2}\right)-4 C_{2} L_{0} L_{1} \operatorname{Pr} N_{b}\right)}{\left(L_{0}^{2}-L_{1}^{2} \operatorname{Pr}^{2} N_{b}^{2}\right)^{2}} .
\end{aligned}
$$

Relations for $L_{15}$ to $L_{18}$ are shown in Box (I).

$$
\begin{aligned}
& L_{19}=\frac{6 L_{5} L_{6}\left(C_{3}\left(L_{1}^{2} \operatorname{Pr}^{2} N_{b}^{2}+L_{0}^{2}\right)-2 C_{2} L_{0} L_{1} \operatorname{Pr} N_{b}\right)}{L_{0}^{2}-L_{1}^{2} \operatorname{Pr}^{2} N_{b}^{2}}, \\
& L_{20}=\frac{3 L_{1}^{2} L_{5}^{2} L_{6} \operatorname{Pr}^{2} N_{b}^{2}}{L_{0}^{6}-L_{0}^{4} L_{1}^{2} \operatorname{Pr}^{2} N_{b}^{2}}, \\
& L_{21}=-\frac{9 L_{1}^{2} L_{6}^{3} \operatorname{Pr}^{2} N_{b}^{2}}{\left(L_{1}^{2} \operatorname{Pr}^{2} N_{b}^{2}-L_{0}^{2}\right)^{3}}, \\
& L_{22}=\frac{12 L_{1}^{2} L_{5} L_{6}^{2} \operatorname{Pr}^{2} N_{b}^{2}}{\left(L_{0}^{3}-L_{0} L_{1}^{2} \operatorname{Pr}^{2} N_{b}^{2}\right)^{2}}, \\
& L_{23}=\frac{L_{15}-L_{17}+L_{18}}{\left(L_{1} \operatorname{Pr} N_{b}+L_{0}\right)\left(L_{1} \operatorname{Pr} N_{b}+2 L_{0}\right)^{2}\left(L_{1} \operatorname{Pr} N_{b}+3 L_{0}\right)}, \\
& L_{24}=\frac{L_{15}+L_{17}+L_{18}}{\left(L_{0}-L_{1} \operatorname{Pr} N_{b}\right)\left(3 L_{0}-L_{1} \operatorname{Pr} N_{b}\right)\left(L_{1} \operatorname{Pr} N_{b}-2 L_{0}\right)^{2}}, \\
& L_{25}=\frac{2\left(L_{16}+L_{19}\right)}{L_{1} \operatorname{Pr} N_{b}\left(L_{0}-L_{1} \operatorname{Pr} N_{b}\right)^{2}\left(L_{1} \operatorname{Pr} N_{b}-2 L_{0}\right)}, \\
& L_{26}=\frac{2\left(L_{16}-L_{19}\right)}{L_{1} \operatorname{Pr} N_{b}\left(L_{1} \operatorname{Pr} N_{b}+L_{0}\right)^{2}\left(L_{1} \operatorname{Pr} N_{b}+2 L_{0}\right)}, \\
& L_{27}=\frac{2\left(L_{15}-L_{18}+2 L_{20}\right)}{L_{1}^{4} \operatorname{Pr} N_{b}^{4}-L_{0}^{2} L_{1}^{2} \operatorname{Pr} N_{b}^{2}},
\end{aligned}
$$

$$
\begin{aligned}
& L_{28}=\frac{L_{21}}{81 L_{1}^{4} \operatorname{Pr}^{4} N_{b}^{4}-9 L_{0}^{2} L_{1}^{2} \operatorname{Pr}^{2} N_{b}^{2}}, \\
& L_{29}=\frac{L_{22}}{16 L_{1}^{4} \operatorname{Pr}^{4} N_{b}^{4}-4 L_{0}^{2} L_{1}^{2} \operatorname{Pr}^{2} N_{b}^{2}}, \\
& L_{30}=\frac{L_{13}+L_{14}}{8 L_{1} \operatorname{Pr} N_{b}\left(L_{0}-2 L_{1} \operatorname{Pr} N_{b}\right)^{2}\left(L_{1} \operatorname{Pr} N_{b}-L_{0}\right)}, \\
& L_{31}=\frac{L_{13}-L_{14}}{8 L_{1} \operatorname{Pr} N_{b}\left(L_{1} \operatorname{Pr} N_{b}+L_{0}\right)\left(2 L_{1} \operatorname{Pr} N_{b}+L_{0}\right)^{2}}, \\
& L_{32}=\frac{L_{7}+L_{8}}{288 L_{0}^{4}}, \\
& L_{33}=\frac{5\left(12 C_{2} L_{5}^{2}-3 L_{7}+L_{8}\right)}{16 L_{0}^{4}}, \\
& L_{34}=\frac{12 C_{2} L_{5}^{2}+3 L_{7}-L_{8}}{8 L_{0}^{3}}, \\
& L_{35}=\frac{5\left(12 C_{3} L_{5}^{2}+3 L_{9}-L_{10}\right)}{16 L_{0}^{4}}, \\
& L_{36}=\frac{L_{9}+L_{10}}{288 L_{0}^{4}}, \\
& L_{37}=\frac{12 C_{3} L_{5}^{2}-3 L_{9}+L_{10}}{8 L_{0}^{3}}, \\
& L_{38}=\frac{L_{6} e^{-h_{1} L_{1} \operatorname{Pr} N_{b}}}{L_{0}^{2} L_{1}^{2} \operatorname{Pr}^{2} N_{b}^{2}-L_{1}^{4} \operatorname{Pr}^{4} N_{b}^{4}}+\frac{h_{1}^{2} L_{5}}{2 L_{0}^{2}}, \\
& L_{39}=\frac{L_{6} e^{-h_{2} L_{1} \operatorname{Pr} N_{b}}}{L_{0}^{2} L_{1}^{2} \operatorname{Pr}^{2} N_{b}^{2}-L_{1}^{4} \operatorname{Pr}^{4} N_{b}^{4}}+\frac{h_{2}^{2} L_{5}}{2 L_{0}^{2}}, \\
& L_{40}=\frac{h_{1} L_{5}}{L_{0}^{2}}-\frac{L_{1} L_{6} \operatorname{Pr} N_{b} e^{-h_{1} L_{1} \operatorname{Pr} N_{b}}}{L_{0}^{2} L_{1}^{2} \operatorname{Pr}^{2} N_{b}^{2}-L_{1}^{4} \operatorname{Pr}^{4} N_{b}^{4}}, \\
& L_{41}=\frac{h_{2} L_{5}}{L_{0}^{2}}-\frac{L_{1} L_{6} \operatorname{Pr} N_{b} e^{-h_{2} L_{1} \operatorname{Pr} N_{b}}}{L_{0}^{2} L_{1}^{2} \operatorname{Pr}^{2} N_{b}^{2}-L_{1}^{4} \operatorname{Pr}^{4} N_{b}^{4}},
\end{aligned}
$$

$$
\begin{aligned}
& L_{15}=\frac{3 L_{0}^{4} L_{6}\left(C_{2}^{2} L_{1}^{2} \operatorname{Pr}^{2} N_{b}^{2}-4 C_{2} C_{3} L_{1} L_{0} \operatorname{Pr} N_{b}+2\left(C_{2}^{2}+C_{3}^{2}\right) L_{0}^{2}\right)}{L_{0}^{2}-L_{1}^{2} \operatorname{Pr}^{2} N_{b}^{2}}, \\
& L_{16}=\frac{6 L_{5} L_{6}\left(C_{2}\left(L_{1}^{2} \operatorname{Pr}^{2} N_{b}^{2}+L_{0}^{2}\right)-2 C_{3} L_{0} L_{1} \operatorname{Pr} N_{b}\right)}{L_{0}^{2}-L_{1}^{2} \operatorname{Pr}^{2} N_{b}^{2}}, \\
& L_{17}=\frac{6 L_{0}^{4} L_{6}\left(2 C_{3} L_{0}-C_{2} L_{1} \operatorname{Pr} N_{b}\right)\left(2 C_{2} L_{0}-C_{3} L_{1} \operatorname{Pr} N_{b}\right)}{L_{0}^{2}-L_{1}^{2} \operatorname{Pr}^{2} N_{b}^{2}}, \\
& L_{18}=\frac{3 L_{0}^{4} L_{6}\left(C_{3}^{2} L_{1}^{2} \operatorname{Pr}^{2} N_{b}^{2}-4 C_{2} C_{3} L_{1} L_{0} \operatorname{Pr} N_{b}+2\left(C_{2}^{2}+C_{3}^{2}\right) L_{0}^{2}\right)}{L_{0}^{2}-L_{1}^{2} \operatorname{Pr}^{2} N_{b}^{2}} .
\end{aligned}
$$




$$
\begin{aligned}
& L_{42}=\frac{1}{4}\left(L_{24} e^{4 h_{1} L_{0}}+L_{25} e^{3 h_{1} L_{0}}+L_{26} e^{h_{1} L_{0}}\right. \\
& \left.+L_{27} e^{2 h_{1} L_{0}}+L_{23}\right) e^{-h_{1}\left(L_{1} \operatorname{Pr} N_{b}+2 L_{0}\right)} \\
& +L_{28} e^{-3 h_{1} L_{1} \operatorname{Pr} N_{b}}+L_{29} e^{-2 h_{1} L_{1} \operatorname{Pr} N_{b}} \\
& +L_{30} e^{2 h_{1} L_{0}-h_{1}\left(2 L_{1} \operatorname{Pr} N_{b}+L_{0}\right)} \\
& +L_{31} e^{-h_{1}\left(2 L_{1} \operatorname{Pr} N_{b}+L_{0}\right)} \\
& +L_{36} \sinh \left(3 h_{1} L_{0}\right)+h_{1} L_{34} \sinh \left(h_{1} L_{0}\right) \\
& +L_{35} \sinh \left(h_{1} L_{0}\right)+L_{32} \cosh \left(3 h_{1} L_{0}\right) \\
& +L_{33} \cosh \left(h_{1} L_{0}\right)+\frac{L_{12} \cosh \left(2 h_{1} L_{0}\right)}{12 L_{0}^{4}} \\
& +\frac{L_{11} \sinh \left(2 h_{1} L_{0}\right)}{L_{0}^{4}}+h_{1} L_{37} \cosh \left(h_{1} L_{0}\right), \\
& L_{43}=\frac{1}{4}\left(L_{24} e^{4 h_{2} L_{0}}+L_{25} e^{3 h_{2} L_{0}}+L_{26} e^{h_{2} L_{0}}\right. \\
& \left.+L_{27} e^{2 h_{2} L_{0}}+L_{23}\right) e^{-h_{2}\left(L_{1} \operatorname{Pr} N_{b}+2 L_{0}\right)} \\
& +L_{28} e^{-3 h_{2} L_{1} \operatorname{Pr} N_{b}}+L_{29} e^{-2 h_{2} L_{1} \operatorname{Pr} N_{b}} \\
& +L_{30} e^{2 h_{2} L_{0}-h_{2}\left(2 L_{1} \operatorname{Pr} N_{b}+L_{0}\right)} \\
& +L_{31} e^{-h_{2}\left(2 L_{1} \operatorname{Pr} N_{b}+L_{0}\right)}+h_{2} L_{34} \sinh \left(h_{2} L_{0}\right. \\
& +L_{35} \sinh \left(h_{2} L_{0}\right)+L_{36} \sinh \left(3 h_{2} L_{0}\right) \\
& +L_{32} \cosh \left(3 h_{2} L_{0}\right)+L_{33} \cosh \left(h_{2} L_{0}\right) \\
& +h_{2} L_{37} \cosh \left(h_{2} L_{0}\right)+\frac{L_{11} \sinh \left(2 h_{2} L_{0}\right)}{L_{0}^{4}} \\
& +\frac{L_{12} \cosh \left(2 h_{2} L_{0}\right)}{12 L_{0}^{4}} \\
& L_{44}=\frac{1}{4}\left(4 L_{0} L_{24} e^{4 h_{1} L_{0}}+3 L_{0} L_{25} e^{3 h_{1} L_{0}}+L_{0} L_{26} e^{h_{1} L_{0}}\right. \\
& \left.+2 L_{0} L_{27} e^{2 h_{1} L_{0}}\right) e^{-h_{1}\left(L_{1} \operatorname{Pr} N_{b}+2 L_{0}\right)} \\
& -3 L_{1} L_{28} \operatorname{Pr} N_{b} e^{-3 h_{1} L_{1} \operatorname{Pr} N_{b}} \\
& -2 L_{1} L_{29} \operatorname{Pr} N_{b} e^{-2 h_{1} L_{1} \operatorname{Pr} N_{b}}+\frac{1}{4}\left(L_{24} e^{4 h_{1} L_{0}}\right. \\
& +L_{25} e^{3 h_{1} L_{0}}+L_{26} e^{h_{1} L_{0}}+L_{27} e^{2 h_{1} L_{0}} \\
& +L_{31}\left(-2 L_{1} \operatorname{Pr} N_{b}-L_{0}\right) e^{-h_{1}\left(2 L_{1} \operatorname{Pr} N_{b}+L_{0}\right)}
\end{aligned}
$$

$$
\begin{aligned}
& \left.+L_{23}\right)\left(-L_{1} \operatorname{Pr} N_{b}-2 L_{0}\right) e^{-h_{1}\left(L_{1} \operatorname{Pr} N_{b}+2 L_{0}\right)} \\
& +L_{30}\left(L_{0}-2 L_{1} \operatorname{Pr} N_{b}\right) e^{2 h_{1} L_{0}-h_{1}\left(2 L_{1} \operatorname{Pr} N_{b}+L_{0}\right)} \\
& +\frac{L_{12} \sinh \left(2 h_{1} L_{0}\right)}{6 L_{0}^{3}}+3 L_{0} L_{32} \sinh \left(3 h_{1} L_{0}\right) \\
& +L_{0} L_{33} \sinh \left(h_{1} L_{0}\right)+L_{34} \sinh \left(h_{1} L_{0}\right) \\
& +h_{1} L_{0} L_{37} \sinh \left(h_{1} L_{0}\right)+\frac{2 L_{11} \cosh \left(2 h_{1} L_{0}\right)}{L_{0}^{3}} \\
& +h_{1} L_{0} L_{34} \cosh \left(h_{1} L_{0}\right)+L_{0} L_{35} \cosh \left(h_{1} L_{0}\right) \\
& +3 L_{0} L_{36} \cosh \left(3 h_{1} L_{0}\right)+L_{37} \cosh \left(h_{1} L_{0}\right) \\
& L_{45}=\frac{1}{4}\left(4 L_{0} L_{24} e^{4 h_{2} L_{0}}+3 L_{0} L_{25} e^{3 h_{2} L_{0}}\right. \\
& \left.+L_{0} L_{26} e^{h_{2} L_{0}}+2 L_{0} L_{27} e^{2 h_{2} L_{0}}\right) e^{-h_{2}\left(L_{1} \operatorname{Pr} N_{b}+2 L_{0}\right)} \\
& +L_{34} \sinh \left(h_{2} L_{0}\right)-3 L_{1} L_{28} \operatorname{Pr} N_{b} e^{-3 h_{2} L_{1} \operatorname{Pr} N_{b}} \\
& -2 L_{1} L_{29} \operatorname{Pr} N_{b} e^{-2 h_{2} L_{1} \operatorname{Pr} N_{b}}+L_{0} L_{33} \sinh \left(h_{2} L_{0}\right) \\
& +\frac{1}{4}\left(L_{24} e^{4 h_{2} L_{0}}+L_{25} e^{3 h_{2} L_{0}}+L_{26} e^{h_{2} L_{0}}\right. \\
& \left.+L_{27} e^{2 h_{2} L_{0}}+L_{23}\right)\left(-L_{1} \operatorname{Pr} N_{b}\right. \\
& \left.-2 L_{0}\right) e^{-h_{2}\left(L_{1} \operatorname{Pr} N_{b}+2 L_{0}\right)}+L_{31}\left(-2 L_{1} \operatorname{Pr} N_{b}\right. \\
& \left.-L_{0}\right) e^{-h_{2}\left(2 L_{1} \operatorname{Pr} N_{b}+L_{0}\right)}+L_{30}\left(L_{0}\right. \\
& \left.-2 L_{1} \operatorname{Pr} N_{b}\right) e^{2 h_{2} L_{0}-h_{2}\left(2 L_{1} \operatorname{Pr} N_{b}+L_{0}\right)} \\
& +3 L_{0} L_{32} \sinh \left(3 h_{2} L_{0}\right)+h_{2} L_{0} L_{37} \sinh \left(h_{2} L_{0}\right) \\
& +h_{2} L_{0} L_{34} \cosh \left(h_{2} L_{0}\right)+L_{0} L_{35} \cosh \left(h_{2} L_{0}\right) \\
& +3 L_{0} L_{36} \cosh \left(3 h_{2} L_{0}\right)+L_{37} \cosh \left(h_{2} L_{0}\right) \\
& +\frac{L_{12} \sinh \left(2 h_{2} L_{0}\right)}{6 L_{0}^{3}}+\frac{2 L_{11} \cosh \left(2 h_{2} L_{0}\right)}{L_{0}^{3}} .
\end{aligned}
$$

\section{Biography}

Safia Akram is an Assistant Professor in National University of Sciences and Technology (MCS), Islamabad, Pakistan. Her field of research is applied mathematics and computationl fluid mechanics. She is recipient of research productive award in years 20102011 and 2011-2012 by Pakistan Council for Science and Technology. Her research articles have been published in international journals. 